\title{
Positron transport in the interstellar medium
}

\author{
P. Jean ${ }^{1}$, W. Gillard ${ }^{1,2,3}$, A. Marcowith ${ }^{4}$, and K. Ferrière ${ }^{5}$ \\ ${ }^{1}$ CESR, Université de Toulouse, CNRS, INSU: 9 avenue du colonel Roche, BP 44346, 31028 Toulouse, France \\ e-mail: Pierre. Jean@cesr.fr \\ 2 KTH, Department of Physics, AlbaNova University Centre, 10691 Stockholm, Sweden \\ 3 The Oskar Klein Centre for Cosmo Particle Physics, AlbaNova, 10691 Stockholm, Sweden \\ ${ }^{4}$ LPTA, CNRS, Université Montpellier II, 34095 Montpellier Cedex 5, France \\ 5 LATT, Université de Toulouse, CNRS: 14 avenue Édouard Belin, 31400 Toulouse, France
}

Received 22 March 2008 / Accepted 22 September 2009

\begin{abstract}
Aims. We seek to understand the propagation mechanisms of positrons in the interstellar medium (ISM). This understanding is a key to determine whether the spatial distribution of the annihilation emission observed in our Galaxy reflects the spatial distribution of positron sources and, therefore, makes it possible to place constraints on the origin of positrons.

Methods. We review the different processes that are likely to affect the transport of positrons in the ISM. These processes fall into three broad categories: scattering off magnetohydrodynamic waves, collisions with particles of the interstellar gas, and advection with large-scale fluid motions. We assess the efficiency of each process and describe its impact on the propagation of positrons. We also develop a model of positron propagation, based on Monte-Carlo simulations, which enable us to estimate the distances traveled by positrons in the different phases of the ISM.

Results. We find that low-energy $(\$ 10 \mathrm{MeV})$ positrons generally have negligible interactions with magnetohydrodynamic waves, insofar as these waves are heavily damped. Positron propagation is mainly controlled by collisions with gas particles. Under these circumstances, positrons can travel very large distances (up to $\sim 30 \mathrm{kpc} / n_{\mathrm{H}, \mathrm{cm}^{-3}}$ for $1 \mathrm{MeV}$ positrons) along magnetic field lines before annihilating.
\end{abstract}

Key words. gamma rays: theory - diffusion - plasmas

\section{Introduction}

Positron annihilation in the Galactic center (GC) region is now a firmly established source of radiation, which has been observed since the early seventies in several balloon and satellite experiments (see von Ballmoos et al. 2003; Jean et al. 2004; Diehl et al. 2006 for reviews). Despite significant progress in observational capabilities, the origin of Galactic positrons remains an open question.

Recent observations of the $511 \mathrm{keV}$ line intensity using the SPI spectrometer onboard the International Gamma-Ray Laboratory (INTEGRAL) observatory have revealed a diffuse emission, distributed in the bulge and the disk of our Galaxy (Knödlseder et al. 2005; Weidenspointner et al. 2006). Observations further indicate that the bulge-to-disk luminosity ratio (hereafter $\mathrm{B} / \mathrm{D})$ of the $511 \mathrm{keV}$ line is rather large $(\approx 3-9)$ compared to the distribution of any candidate source. Under the hypothesis that positrons annihilate close to their sources, the spatial distribution of the annihilation emission should reflect the spatial distribution of the positron sources. In this view, the large $\mathrm{B} / \mathrm{D}$ ratio could be explained by sources belonging to the old stellar population (Knödlseder et al. 2005) and a disk emission, partly or totally, attributed to the radioactive decay of ${ }^{26} \mathrm{Al}$ and ${ }^{44} \mathrm{Ti}$ produced in massive stars. The most recent SPI analysis covering more than 4 years of data shows hints of a longitudinal asymmetry in the spatial distribution of the $511 \mathrm{keV}$ line emission produced in the inner part of the Galactic disk (Weidenspointner et al. 2008). A similar asymmetry is observed in the distribution of low-mass X-ray binaries emitting at high energies, suggesting that these objects might be the dominant sources of positrons. This conclusion is contingent upon the hypothesis that positrons annihilate close to their sources, a hypothesis that should be called into question.

Several authors have argued that positrons annihilate in the vicinity of their production sites, based on the assumption that particles propagate according to the so-called Bohm diffusion, i.e., with a mean free-path equal to their Larmor radius (Boehm et al. 2004; Wang 2006). This implicitly supposes that magnetic fluctuations in the ISM are strongly tangled on all scales, as is likely the case near strong shocks in supernova remnants. In reality, given the complexity and the variety of thermodynamical phases in the ISM, Bohm diffusion probably overestimates particle confinement (Parizot et al. 2004a). Jean et al. (2006) estimated the distances traveled by positrons using a model combining a quasi-linear diffusion theory of wave-particle interactions at high energy and an approximate propagation model including collisions with the ambient ISM matter at low energy. A preliminary model of the gas content in the Galactic bulge (a more complete description of the gas spatial distribution in this region is presented in Ferrière et al. 2007) was then added to the propagation model. The authors concluded that $\mathrm{MeV}$ positrons injected by radioactive processes into the bulge cannot escape from it and that a single source releasing positrons in the bulge might have difficulties accounting for the observed spatial extent of the annihilation emission. Prantzos (2006) proposed a solution to explain the large B/D ratio measured with SPI, in which 
positrons produced by type Ia supernovae in the old disk are transported along magnetic field lines into the bulge. Cheng et al. (2006) suggested that positrons in the bulge could originate from the decay of $\pi^{+}$produced in high-energy $p p$ collisions. In this work, the energetic protons would be shock-accelerated when a star is tidally disrupted in the accretion disk of Sagittarus $\mathrm{A}^{*}$ $\left(\right.$ Sgr A* $^{*}$. Since high-energy $(>30 \mathrm{MeV})$ positrons take as long as $\sim 10^{7}$ years to cool down, they should be able to propagate far away from $\mathrm{Sgr} \mathrm{A}^{*}$ and to fill the Galactic bulge. In order to explain the annihilation emission in the bulge, Totani (2006) proposed a scenario in which $\mathrm{MeV}$ positrons were produced in the accretion disk of $\mathrm{Sgr} \mathrm{A}^{*} \sim 10^{7}$ years ago. These positrons would have filled the bulge while being transported by large-scale outflows $\left(\sim 100 \mathrm{~km} \mathrm{~s}^{-1}\right)$. All these studies invoked positron transport to explain the observed annihilation emission, but they did not include any detailed examination of the physical processes that could contribute to it.

More recently, Higdon et al. (2009) discussed the role of magnetohydrodynamical (MHD) fluctuations in positron transport in the different ISM phases. They further estimated the positron mean free path using a phenomenological model of $\mathrm{MeV}$ electron transport in interplanetary turbulence, especially in the ionized phases where turbulence is undamped down to small scales (see appendix). However, the model used to derive the positron mean free-path is strictly valid for interplanetary plasmas, and the nature of ISM turbulence is likely different. The model developed by Higdon et al. (2009) is up to now the most valuable attempt to account for the propagation of low-energy positrons in the ISM. Here, rather than considering a particular turbulent model, we review the transport processes that govern the positron mean free path.

We discuss three different regimes of positron transport in the Galaxy, namely, the regime dominated by scattering off MHD waves (Sect. 2), the regime dominated by collisions with particles of the interstellar gas (Sect. 3) and a regime of advection with large-scale fluid motion (Sect. 4). Based on the quantitative results obtained in each section, we infer the relevance and the importance of the respective regimes. In Sect. 5, we summarize our study and discuss its possible implications for positron transport at Galactic scales.

\section{Scattering off magnetohydrodynamic waves}

In Sect. 2.1, we present the condition for wave-particle resonance, restricting our analysis to magnetohydrodynamic (MHD) waves. We further discuss the effective value of the Alfvén speed in the neutral phases of the ISM. In Sect. 2.2, we present the properties of the MHD wave cascades initiated at large spatial scales, e.g., by the differential rotation of the Galaxy or by the explosion of supernovae. In Sect. 2.2.1, we present the different energy transfer times associated with the different type of cascades that likely developped in the ISM. In Sect. 2.2.2, we discuss the collisional and collisionless damping mechanisms of MHD waves as well as the smallest scales of the MHD cascades in the different ISM phases. Only a brief summary is given there; the technical derivation is postponed to Appendix A. In Sect. 2.3, we discuss the global effect on positron transport of MHD waves injected at large scales. In Sect. 2.4, we consider the local effect of magnetic fluctuations generated by the streaming instability, which can survive down to the small scales of low-energy positrons.

Throughout Sect. 2, we assume, for simplicity, that the interstellar gas contains only hydrogen.

\subsection{Positron resonance with $M H D$ waves}

In a medium with non-vanishing magnetic field, wave-particle interactions proceed through the Landau-synchrotron resonance condition expressed as (Melrose 1986):

$\omega-k_{\|} v_{\|}=\ell \Omega_{\mathrm{se}}$,

where $\omega$ is the wave frequency, $k_{\|}$the component of the wave vector along magnetic field lines, $v_{\|}$the particle velocity along field lines, $\ell$ an integer, and $\Omega_{\mathrm{se}}=\Omega_{\mathrm{ce}} / \gamma$ the synchrotron frequency of the particle (a positron $\mathrm{e}^{+}$in the case at hand, hence the subscript e), with $\gamma$ the particle Lorentz factor, $\Omega_{\mathrm{ce}}=$ $\left(e B / m_{\mathrm{e}} c\right)$ the particle cyclotron frequency, and $B$ the magnetic field strength.

In this work, we consider only MHD waves, more specifically, shear Alfvén waves and fast magnetosonic waves as these waves are a major part of the magnetic fluctuations that pervade the ISM (Lithwick \& Goldreich 2001). We do not consider slow waves separately, because the dynamics of the slow wave cascade were shown to be entirely controlled by the Alfvén wave cascade (Lithwick \& Goldreich 2001) and the slow wave spectrum is basically the same as the Alfvén wave spectrum. Following Yan \& Lazarian (2004), we assume that the transport of charged particles is governed by either Alfvén waves or fast magnetosonic waves. Note that higher-frequency waves are potentially important as well, as they can easily fulfill the above resonance condition with positrons produced by radioactive decay (energy $\lesssim 1 \mathrm{MeV}$ ). However, whistler waves, the most interesting waves in this frequency domain, are right-handed polarized and, therefore, cannot be in resonance with positrons. Higdon et al. (2009) have discussed the possible role of selfgenerated whistler waves. We will come back to this possibility in Sect. 2.4. The possible effects of large-scale compressible motion will be examined in Sect. 4 .

For the waves of interest here, it can be shown that $\omega \ll$ $\Omega_{\mathrm{se}}{ }^{1}$, so we are entitled to neglect $\omega$ in Eq. (1), except in the particular case $\ell=0$. The case $\ell=0$ corresponds to the socalled Cherenkov resonance, in which the particle interacts with a zero-frequency wave in a frame moving at velocity $v_{\|}$. In that case, Eq. (1) reduces to $\omega=k_{\|} v_{\|}$. The Cherenkov resonance can be important for magnetosonic waves, which have a perturbed magnetic field component parallel to the background magnetic field.

Here, we consider only the dominant harmonics $\ell= \pm 1$. With $\omega \ll \Omega_{\text {se }}$, Eq. (1) can then be recast into the form

$k_{\|} r_{\mathrm{L}}^{\prime} \cos \alpha \simeq \pm 1$,

where $\alpha$ is the particle pitch angle (angle between the particle velocity and the local magnetic field $), r_{\mathrm{L}}^{\prime}=\left(v / \Omega_{\mathrm{se}}\right)=(p c / e B)$ is the particle gyroradius divided by $\sin \alpha$ (simply referred to as the gyroradius in the following), and $p=\gamma m_{\mathrm{e}} v$ is the particle momentum. Equation (2) expresses the resonance condition in terms of the particle gyroradius. It can be rewritten in terms of the particle momentum as

$p= \pm \frac{e B}{c k_{\|} \cos \alpha}$

or in terms of the particle kinetic energy, $E_{k}$, as

$E_{k}=\left(m_{\mathrm{e}} c^{2}\right)\left[\sqrt{\left(\frac{e B}{m_{\mathrm{e}} c^{2} k_{\|} \cos \alpha}\right)^{2}+1}-1\right]$.

\footnotetext{
1 According to Eq. (5), this condition is automatically satisfied for positrons with $\gamma \ll m_{\mathrm{p}} / m_{\mathrm{e}}$, corresponding to energies $\ll 0.94 \mathrm{GeV}$.
} 
Table 1. Physical parameters of the different ISM phases.

\begin{tabular}{lccccccc}
\hline \hline ISM phase & $\begin{array}{c}T \\
(\mathrm{~K})\end{array}$ & $\begin{array}{c}B \\
(\mu \mathrm{G})\end{array}$ & $\begin{array}{c}n_{\mathrm{H}} \\
\left(\mathrm{cm}^{-3}\right)\end{array}$ & $f_{\text {ion }}$ & $\begin{array}{c}n_{\mathrm{i}} \\
\left(\mathrm{cm}^{-3}\right)\end{array}$ & $\begin{array}{c}k_{\| \max } \\
\left(10^{-9} \mathrm{~cm}^{-1}\right)\end{array}$ & $\begin{array}{c}E_{\mathrm{k}, \min } \\
(\mathrm{keV})\end{array}$ \\
\hline HIM (low $B)$ & $10^{6}$ & 2 & $0.005-0.01$ & 1 & $0.005-0.01$ & $3.1-4.4$ & $35-18$ \\
HIM (high $B)$ & $10^{6}$ & 20 & $0.005-0.01$ & 1 & $0.005-0.01$ & $3.1-4.4$ & $1500-950$ \\
WIM & 8000 & 5 & $0.2-0.5$ & $0.6-0.9$ & $0.12-0.45$ & $15-29$ & $9.5-2.5$ \\
WNM & $6000-10000$ & 5 & $0.2-0.5$ & $0.007-0.05$ & $0.0014-0.025$ & $1.6-6.9$ & $540-45$ \\
CNM & $50-100$ & 6 & $20-50$ & $4 \times 10^{-4}-10^{-3}$ & $0.008-0.05$ & $3.9-9.8$ & $175-32$ \\
MM & $10-20$ & $8.5-850$ & $10^{2}-10^{6}$ & $\lesssim 10^{-4}$ & & $\lesssim 4.4$ & $\gtrsim 265$ \\
\hline
\end{tabular}

Note to the table: The different ISM phases are molecular medium (MM), cold neutral medium (CNM), warm neutral medium (WNM), warm ionized medium (WIM) and hot ionized medium (HIM). $T$ is the temperature, $B$ the magnetic field strength, $n_{\mathrm{H}}$ the hydrogen density, $f_{\text {ion }}=$ $n_{\mathrm{i}} /\left(n_{\mathrm{i}}+n_{\mathrm{n}}\right)$ the ionization fraction, $n_{\mathrm{i}}$ the ion density, $k_{\| \max }$ the maximum parallel wavenumber of Alfvén waves (right-hand side of Eq. (12)), and $E_{\mathrm{k}, \text { min }}$ the minimum kinetic energy required for positrons to interact resonantly with Alfvén waves (right-hand side of Eq. (13)). Here, we assume a pure-hydrogen gas, for which $n_{\mathrm{i}}=f_{\text {ion }} n_{\mathrm{H}}$ and $n_{\mathrm{n}}=\left(1-f_{\text {ion }}\right) n_{\mathrm{H}}$.

Roughly speaking, MHD waves can exist only at frequencies lower than the proton cyclotron frequency, $\Omega_{\mathrm{cp}}=\left(\mathrm{eB} / \mathrm{m}_{\mathrm{p}} \mathrm{c}\right)$ :

$\omega \leq \Omega_{\mathrm{cp}}$.

Either they are damped by collisional effects (mainly viscous friction and ion-neutral collisions) at low frequencies (see Sect. A.1) or, if they manage to survive collisional effects, then at frequencies approaching $\Omega_{\mathrm{cp}}$, they are heavily damped by Landau damping due to thermal protons (see Sect. A.2).

For Alfvén waves ( $\omega=V_{\mathrm{A}} k_{\|}$, with $V_{\mathrm{A}}$ the Alfvén speed), Eq. (5) is equivalent to

$k_{\|} \leq \frac{\Omega_{\mathrm{cp}}}{V_{\mathrm{A}}}$,

which, in view of the resonance conditions, Eqs. (3) and (4), implies a threshold on the positron momentum (obtained for $|\cos \alpha|=1)$ :

$p \geq m_{\mathrm{p}} V_{\mathrm{A}}$,

and a threshold on the positron kinetic energy:

$E_{k} \geq\left(m_{\mathrm{e}} c^{2}\right)\left[\sqrt{\left(\frac{m_{\mathrm{p}} V_{\mathrm{A}}}{m_{\mathrm{e}} c}\right)^{2}+1}-1\right]$.

For fast magnetosonic waves $\left(\omega=V_{\mathrm{F}}(\Theta) k\right.$, with $\Theta$ the angle between the wave vector and the magnetic field, $V_{\mathrm{F}}$ the phase speed of the fast mode, comprised between $V_{\mathrm{F}}(0)=\max \left(V_{\mathrm{A}}, C_{\mathrm{s}}\right)$ and $V_{\mathrm{F}}(\pi / 2)=V_{\mathrm{ms}} \equiv \sqrt{V_{\mathrm{A}}^{2}+C_{\mathrm{S}}^{2}}$, and $C_{\mathrm{s}}$ the adiabatic sound speed), Eq. (5) leads to $k \leq \Omega_{\mathrm{cp}} / V_{\mathrm{F}}(\Theta)$ and, hence, $p \geq m_{\mathrm{p}} V_{\mathrm{F}}(\Theta) / \cos \Theta$. Since the functions $V_{\mathrm{F}}(\Theta)$ and $V_{\mathrm{F}}(\Theta) / \cos \Theta$ reach their minimum values at $\Theta=0$, the absolute requirement on the wavenumber reads

$k \leq \frac{\Omega_{\mathrm{cp}}}{V_{\mathrm{F}}(0)}$,

and the thresholds on the positron momentum and kinetic energy (again obtained for $|\cos \alpha|=1$ ) are given by

$p \geq m_{\mathrm{p}} V_{\mathrm{F}}(0)$

and

$E_{k} \geq\left(m_{\mathrm{e}} c^{2}\right)\left[\sqrt{\left(\frac{m_{\mathrm{p}} V_{\mathrm{F}}(0)}{m_{\mathrm{e}} c}\right)^{2}+1}-1\right]$,

respectively.
The question now is what expression should be used for the Alfvén speed. In a fully ionized medium, the Alfvén speed is simply $V_{\mathrm{A}}=B / \sqrt{4 \pi \rho_{\mathrm{i}}}$, with $\rho_{\mathrm{i}}$ the ion mass density. However, in a partially ionized medium, the relevant Alfvén speed depends on the degree of coupling between ions and neutrals. If the ion-neutral and neutral-ion collision frequencies, $v_{\mathrm{in}}$ and $v_{\mathrm{ni}}$, are much greater than the wave frequency, $\omega$, then ions and neutrals are very well coupled through ion-neutral collisions, and as a result, an Alfvén wave will set the entire fluid (ions + neutrals) into motion. In that case, one should use the total Alfvén speed, $V_{\mathrm{A}, \text { tot }}=B / \sqrt{4 \pi \rho_{\text {tot }}}$, with $\rho_{\text {tot }}=\rho_{\mathrm{i}}+\rho_{\mathrm{n}}$ the total (ion + neutral) mass density. In contrast, if the ion-neutral and neutral-ion collision frequencies are much smaller than the wave frequency, then ions and neutrals are no longer coupled, and an Alfvén wave will set the ions alone in motion. In that case, one should use the ionic Alfvén speed, $V_{\mathrm{A}, \mathrm{i}}=B / \sqrt{4 \pi \rho_{\mathrm{i}}}$, as in a fully ionized medium.

In an atomic medium with temperature $T \lesssim 100 \mathrm{~K}$, the ion-neutral and neutral-ion collision frequencies are given by $v_{\mathrm{in}} \simeq\left(1.6 \times 10^{-9} \mathrm{~cm}^{3} \mathrm{~s}^{-1}\right) n_{\mathrm{n}}$ and $v_{\mathrm{ni}} \simeq\left(1.6 \times 10^{-9} \mathrm{~cm}^{3} \mathrm{~s}^{-1}\right) n_{\mathrm{i}}$, where $n_{\mathrm{n}}$ and $n_{\mathrm{i}}$ are the neutral and ion number densities, respectively (Osterbrock 1961). At high temperature, the collision frequencies increase as $\sqrt{T}$ (Braginskii 1965; Shull \& Draine 1987); assuming an effective cross section for $\mathrm{H}^{-} \mathrm{H}^{+}$collisions $\sim 10^{-14} \mathrm{~cm}^{2}$ (Wentzel 1974), we find that, for $T \gtrsim 140 \mathrm{~K}$, $v_{\text {in }} \sim\left(1.4 \times 10^{-9} \mathrm{~cm}^{3} \mathrm{~s}^{-1}\right) \sqrt{T / 100 \mathrm{~K}} n_{\mathrm{n}}$ and $v_{\mathrm{ni}} \sim(1.4 \times$ $\left.10^{-9} \mathrm{~cm}^{3} \mathrm{~s}^{-1}\right) \sqrt{T / 100 \mathrm{~K}} n_{\mathrm{i}}$. In a molecular medium, the collision frequencies are $v_{\text {in }} \simeq\left(2.1 \times 10^{-9} \mathrm{~cm}^{3} \mathrm{~s}^{-1}\right) n_{\mathrm{n}}$ and $v_{\mathrm{ni}} \simeq$ $\left(2.1 \times 10^{-9} \mathrm{~cm}^{3} \mathrm{~s}^{-1}\right) n_{\mathrm{i}}$ (Osterbrock 1961). For comparison, the frequency of a resonant Alfvén wave is given by $\omega=V_{\mathrm{A}} k_{\|}$with, according to Eq. (2), $k_{\|} \gtrsim 1 / r_{\mathrm{L}}^{\prime}=(e B / p c)$, i.e., $\omega \gtrsim(6.6 \times$ $\left.10^{-5} \mathrm{~s}^{-1}\right)\left(B_{\mu \mathrm{G}}^{2} / \sqrt{n_{\mathrm{cm}^{-3}}}\right)(p c)_{\mathrm{MeV}}^{-1}$, where $n$ is the relevant number density (e.g., $n=n_{\mathrm{i}}+n_{\mathrm{n}}$ if $v_{\mathrm{in}}, \nu_{\mathrm{ni}} \gg \omega$ and $n=n_{\mathrm{i}}$ if $\left.v_{\text {in }}, v_{\text {ni }} \ll \omega\right)$. From this, it follows that for typical interstellar conditions (see Table 1), $v_{\mathrm{in}}, v_{\mathrm{ni}} \ll \omega$ up to positron energies of at least $1 \mathrm{GeV}$. Hence, in the present context, the relevant Alfvén speed is $V_{\mathrm{A}, \mathrm{i}}=B / \sqrt{4 \pi \rho_{\mathrm{i}}}$, not only in the ionized phases, but also in the so-called neutral (i.e., atomic and molecular) phases.

\section{Alfvén waves}

With the above statements in mind, the requirement on the parallel wavenumber of Alfvén waves, Eq. (6), can be rewritten as

$k_{\|} \leq \frac{\sqrt{4 \pi} e}{\sqrt{m_{\mathrm{p}}} c} \sqrt{n_{\mathrm{i}}}=\left(4.4 \times 10^{-8} \mathrm{~cm}^{-1}\right) \sqrt{n_{\mathrm{i}, \mathrm{cm}^{-3}}}$. 
The corresponding condition on the positron kinetic energy, Eq. (8), becomes

$$
E_{k} \geq(511 \mathrm{keV})\left[\sqrt{1.8 \times 10^{-4} \frac{B_{\mu \mathrm{G}}^{2}}{n_{\mathrm{i}, \mathrm{cm}^{-3}}}+1}-1\right] .
$$

The above expressions were obtained on the assumption that the only ion present in the ISM is $\mathrm{H}^{+}$. To account for the presence of other ions, it suffices, to a good approximation, to replace $n_{\mathrm{i}}$ by $n_{\mathrm{H}}+4 n_{\mathrm{He}}$ with $n_{\mathrm{He}} \simeq 0.1 n_{\mathrm{H}}$ in the hot phase and by $n_{\mathrm{H}^{+}}+$ $12 n_{\mathrm{C}, \text { gas }}$ with $n_{\mathrm{C}, \text { gas }} \simeq 1.4 \times 10^{-4} n_{\mathrm{H}}$ (Cardelli et al. 1996) in the atomic phases.

The maximum parallel wavenumber, $k_{\| \max }$, and the minimum kinetic energy, $E_{\mathrm{k}, \min }$, given by the right-hand sides of Eqs. (12) and (13), respectively, are listed in Table 1 for the different phases of the ISM. Also listed in Table 1 are the estimated temperature, $T$, magnetic field strength, $B$, hydrogen density, $n_{\mathrm{H}}$, and ionization fraction, $f_{\text {ion }}=n_{\mathrm{i}} /\left(n_{\mathrm{i}}+n_{\mathrm{n}}\right)$ of the different phases. The values of $T, n_{\mathrm{H}}$ and $f_{\text {ion }}$ are taken from the review paper of Ferrière (2001). For $B$, we adopt the value of $5 \mu \mathrm{G}$ inferred from rotation measure studies (e.g. Rand \& Kulkarni 1989; Ohno \& Shibata 1993) for the warm phases, the value of $6 \mu \mathrm{G}$ inferred from Zeeman splitting measurements (Heiles \& Troland 2005) for the cold phase, the relation $B \propto \sqrt{n_{\mathrm{H}}}$ normalized to $B=85 \mu \mathrm{G}$ at $n_{\mathrm{H}}=10^{4} \mathrm{~cm}^{-3}$ (Crutcher 1999) for molecular clouds, and the two extreme values of $2 \mu \mathrm{G}$ and $20 \mu \mathrm{G}$ for the hot phase. The lower value pertains to the standard scenario in which the hot gas is generated by stellar winds and supernova explosions, which sweep up the ambient magnetic field lines and evacuate them from the hot cavities. The higher value pertains to an alternative scenario in which largescale highly turbulent MHD fluctuations produce magnetic fields above equipartition with the local thermal pressure (Bykov 2001; Parizot et al. 2004b; and references therein).

From Table 1, it emerges that the maximum parallel wavenumber of Alfvén waves is typically a few $10^{-9} \mathrm{~cm}^{-1}$, close to the largest wavenumber, $k_{\mathrm{L}} \simeq 10^{-9} \mathrm{~cm}^{-1}$, of the electron density power spectrum inferred from interstellar scintillation (Armstrong et al. 1995). Furthermore, the minimum kinetic energy required for positrons to interact resonantly with Alfvén waves varies from a few $\mathrm{keV}$ (in the warm ionized medium) to a few hundreds of $\mathrm{keV}$ (in regions with large Alfvén speeds, namely, in molecular clouds and possibly in the warm neutral and hot ionized media). For comparison, positrons produced by radioactive decay are injected into the ISM with typical kinetic energies $\sim 1 \mathrm{MeV}$. This means that positrons from radioactive decay can interact resonantly with Alfvén waves only over a restricted energy range. This range is particularly narrow in regions with large Alfvén speeds, such as molecular clouds (where $V_{\mathrm{A}, \mathrm{i}} \gtrsim 185 \mathrm{~km} \mathrm{~s}^{-1}$ ); it can even vanish in the hot phase if the magnetic field is as strong as $20 \mu \mathrm{G}$ (implying $V_{\mathrm{A}, \mathrm{i}} \gtrsim 436 \mathrm{~km} \mathrm{~s}^{-1}$ ). In contrast, the resonant range extends over at least two orders of magnitude in the warm ionized medium, where the ion density is highest (and $V_{\mathrm{A}, \mathrm{i}} \simeq(16-31) \mathrm{km} \mathrm{s}^{-1}$ ). The reason why resonant interactions with Alfvén waves are no longer possible below $E_{\mathrm{k}, \mathrm{min}}$ is because the Larmor radius has become smaller than the smallest possible scale of existing Alfvén waves.

\section{Fast magnetosonic waves}

The numerical expressions and values of the wavenumber and kinetic energy thresholds can be obtained in the same manner as for Alfvén waves, with the two following differences: First, the wavenumber threshold (Eq. (9)) applies to the total wavenumber, as opposed to the parallel wavenumber. Second, the speed entering the expressions of the thresholds is the phase speed of the fast mode for parallel propagation, $V_{\mathrm{F}}(0)$, as opposed to the Alfvén speed, $V_{\mathrm{A}}$. In practice, however, the second difference is only formal, except in the hot low- $B$ phase. Indeed, $V_{\mathrm{F}}(0)=\max \left(V_{\mathrm{A}}, C_{\mathrm{s}}\right)$, and $\max \left(V_{\mathrm{A}}, C_{\mathrm{s}}\right)=V_{\mathrm{A}}$ in all the ISM phases, except in the hot low- $B$ phase, where $\max \left(V_{\mathrm{A}}, C_{\mathrm{s}}\right)=C_{\mathrm{s}} \simeq 166 \mathrm{~km} \mathrm{~s}^{-1}$. In consequence, the values of the maximum wavenumber, $k_{\max }$, and the minimum kinetic energy, $E_{\mathrm{k}, \min }$, are those listed in Table 1, except in the hot low- $B$ phase, for which Eqs. (9) and (11) lead to $k_{\max } \simeq 1.2 \times 10^{-9} \mathrm{~cm}^{-1}$ and $E_{\mathrm{k}, \min } \simeq 220 \mathrm{keV}$, respectively. The latter value is roughly an order of magnitude larger than for Alfvén waves, which significantly narrows down the energy range over which positrons from radioactive decay can interact resonantly with fast magnetosonic waves.

The Cherenkov resonance (for $\ell=0$ ) occurs when $\omega=$ $k_{\|} v_{\|}$, i.e., at a wave propagation angle $\Theta_{\mathrm{T}}$ such that $V_{\mathrm{F}}\left(\Theta_{\mathrm{T}}\right)=$ $v_{\|} \cos \Theta_{\mathrm{T}}$, independent of the wavenumber. Since $V_{\mathrm{F}}\left(\Theta_{\mathrm{T}}\right)$ never departs from the fast magnetosonic speed, $V_{\mathrm{ms}}$, by more than a factor $\sqrt{2}$, this expression is approximately equivalent to $\cos \Theta_{\mathrm{T}}=V_{\mathrm{ms}} / v_{\|}$. The Cherenkov resonance requires that the wave at propagation angle $\Theta_{\mathrm{T}}$ exist and not be damped by a collisional or collisionless process (see next subsections).

Let us re-emphasize that the above results should be considered only as rough estimates. As we will now see, both Alfvén and fast magnetosonic waves are subject to various damping processes in the ISM.

\subsection{MHD wave cascades}

\subsubsection{Energy transfer timescales}

We consider both Alfvén waves and fast magnetosonic waves as parts of turbulent cascades. The main sources of turbulence able to counterbalance the dissipation mechanisms expected in the ISM are the magnetorotational instability driven by the differential rotation of the Galaxy and the explosion of supernovae (Mac Low \& Klessen 2004). Both mechanisms release energy at large scales. In the rest of the paper, we adopt $L_{\mathrm{inj}}=100 \mathrm{pc}$ for the injection scale. This does not preclude the possibility of injecting magnetic fluctuations at smaller scales; an issue discussed in Sect. 2.4.

\section{Alfvén wave cascade}

The most recent developments in MHD turbulence theory explain the energy cascade towards smaller scales by the distortion of oppositely travelling Alfvén wave packets (e.g., Lithwick \& Goldreich 2001). The kinematics of the interactions produce a highly anisotropic cascade, which redistributes most of the energy in the perpendicular scales ${ }^{2}$. We will return to this important question in Sect. 2.3.

The transfer time of the Alfvén wave cascade, $\tau_{\mathrm{A}}$, corresponds to the wave packet crossing time along the mean magnetic field:

$$
\tau_{\mathrm{A}}=\frac{1}{V_{\mathrm{A}} k_{\|}} .
$$

\footnotetext{
2 Throughout this paper, the perpendicular and parallel directions are taken with respect to the mean magnetic field direction.
} 
For reference, when $L_{\mathrm{inj}}=100 \mathrm{pc}$, one has

$\tau_{\mathrm{A}}=\left(1.4 \times 10^{15} \mathrm{~s}\right) \frac{\sqrt{n_{\mathrm{cm}}-3}}{B_{\mu \mathrm{G}}} \frac{1}{k L_{\mathrm{inj}} \cos \Theta}$,

where $n$ is the relevant number density (in a fully ionized medium, $n=n_{\mathrm{i}}$, while in a partially ionized medium, $n=n_{\mathrm{i}}+n_{\mathrm{n}}$ if $v_{\mathrm{in}}, v_{\mathrm{ni}} \gg \omega$ and $n=n_{\mathrm{i}}$ if $\left.v_{\mathrm{in}}, v_{\mathrm{ni}} \ll \omega\right)$.

\section{Fast magnetosonic wave cascade}

The transfer time of the fast magnetosonic wave cascade, $\tau_{\mathrm{F}}$, can be written as

$\tau_{\mathrm{F}}=\frac{\omega}{k^{2} \delta v_{k}^{2}}=\frac{V_{\mathrm{F}} L_{\mathrm{inj}}^{1 / 2}}{\delta v_{\mathrm{inj}}^{2}} k^{-1 / 2}$,

where $\delta v_{k}$ is the turbulent velocity at scale $k^{-1}, \delta v_{\text {inj }}$ is the turbulent velocity at the injection scale, $L_{\mathrm{inj}}$, and, as before, $V_{\mathrm{F}}$ is the phase speed of the fast mode (Yan \& Lazarian 2004). In writing the second identity, we assumed a Kraichnan spectrum $\left(\mathrm{d} E / \mathrm{d} k \propto k^{-3 / 2}\right.$ or $\left.\delta v_{k} \propto k^{-1 / 4}\right)$. For our numerical estimates, we adopt again $L_{\text {inj }}=100 \mathrm{pc}$ and we set both $\delta v_{\text {inj }}$ and $V_{\mathrm{F}}$ to the fast magnetosonic speed, $V_{\mathrm{ms}}=\sqrt{V_{\mathrm{A}}^{2}+C_{\mathrm{s}}^{2}}$ (as mentioned earlier, $V_{\mathrm{F}}$ does not depart from $V_{\mathrm{ms}}$ by more than a factor $\sqrt{2}$ ), whereupon we find for $L_{\mathrm{inj}}=100 \mathrm{pc}$

$\tau_{\mathrm{F}}=\left(1.4 \times 10^{15} \mathrm{~s}\right) \frac{\sqrt{n_{\mathrm{cm}^{-3}}}}{B_{\mu \mathrm{G}} \sqrt{1+C_{\mathrm{s}}^{2} / V_{\mathrm{A}}^{2}}}\left(k L_{\mathrm{inj}}\right)^{-1 / 2}$,

with the density $n$ defined as for the Alfvén wave cascade.

For both Alfvén and fast magnetosonic waves, the dominant damping process depends on the wavelength (or inverse wavenumber) compared to the proton collisional mean free-path,

$\lambda_{\mathrm{p}}=v_{\mathrm{p}} \tau_{\mathrm{p}} \simeq\left(3.5 \times 10^{13} \mathrm{~cm}\right) \frac{T_{\mathrm{p}, \mathrm{eV}}^{2}}{n_{\mathrm{e}, \mathrm{cm}^{-3}} \Lambda}$,

where $v_{\mathrm{p}}=\sqrt{3 k_{\mathrm{B}} T_{\mathrm{p}} / m_{\mathrm{p}}}$ is the proton rms velocity, $\tau_{\mathrm{p}}$ the proton collision time, $n_{\mathrm{e}}$ the electron density, and $\Lambda$ the Coulomb logarithm, given by

$\Lambda=23.4-0.5 \ln n_{\mathrm{e}, \mathrm{cm}^{-3}}+1.5 \ln T_{\mathrm{e}, \mathrm{eV}}, \quad$ for $T_{\mathrm{e}}<50 \mathrm{eV}$

and

$\Lambda=25.3-0.5 \ln n_{\mathrm{e}, \mathrm{cm}^{-3}}+\ln T_{\mathrm{e}, \mathrm{eV}}, \quad$ for $T_{\mathrm{e}}>50 \mathrm{eV}$

(Braginskii 1965). Waves with $k^{-1}>\lambda_{\mathrm{p}}$ are basically collisional and, therefore, affected by collisional damping (see Sect. A.1), whereas waves with $k^{-1}<\lambda_{\mathrm{p}}$ are basically collisionless and affected by collisionless damping (see Sect. A.2).

As we will see below, in all the cases considered here, $L_{\text {inj }}>\lambda_{\mathrm{p}}$, which means that both turbulent cascades start in the collisional range. The wave energy is then transferred to smaller scales up to the point where the wave damping rate, $\Gamma$, becomes equal to the transfer rate. In other words, the turbulent cascades are cut off at a wavenumber $k_{\text {cut }}$ such that

$\Gamma \tau_{\mathrm{A}}=1$

for the Alfvén cascade and

$\Gamma \tau_{\mathrm{F}}=1$ for the fast magnetosonic cascade. If the collisional damping rate, $\Gamma_{\text {coll }}$, is high enough that $\Gamma_{\text {coll }} \tau_{\mathrm{A}}=1$ at a scale larger than $\lambda_{\mathrm{p}}$, then the Alfvén cascade is cut off by collisional damping at that scale. On the other hand, if $\Gamma_{\text {coll }} \tau_{\mathrm{A}}<1$ down to $\lambda_{\mathrm{p}}$, then the Alfvén cascade proceeds down to the collisionless range and is eventually cut off by collisionless damping at a scale smaller than $\lambda_{\mathrm{p}}$. The situation is similar for the fast magnetosonic cascade.

\subsubsection{Damping and cutoff}

In this section, we summarize the investigation presented in Appendix A on the dominant damping processes of the Alfvén and fast magnetosonic wave cascades in the different ISM phases.

In the mostly neutral, atomic and molecular phases of the ISM, the Alfvén and fast magnetosonic wave cascades are, regardless of their origin, both cut off by ion-neutral collisions at scales larger than the proton mean free-path, $\lambda_{\mathrm{p}}$, i.e., in the collisional regime - and thus at scales considerably larger than the Larmor radii of interstellar positrons. In consequence, positrons will find no Alfvén or fast magnetosonic waves from an MHD cascade to resonantly interact with (see also Higdon et al. 2009).

The situation is completely different in the ionized phases of the ISM. There, the Alfvén wave cascade develops with insignificant (collisional) damping down to $\lambda_{\mathrm{p}}$. It then enters the collisionless range, where it is eventually cut off by linear Landau damping around the proton inertial length. Thus, the extended inertial range of the Alfvén wave cascade leaves some room for possible resonant interactions with positrons. The fast magnetosonic wave cascade, for its part, suffers strong collisional (viscous) damping. In the warm ionized phase, this damping is sufficient to destroy the cascade (with the possible exception of quasi-parallel waves) before it enters the collisionless range. In the hot ionized phase, the cascade manages to reach the collisionless range, but it is then quickly destroyed by linear Landau damping (again with the possible exception of quasi-parallel waves). Altogether, no fast magnetosonic waves from an MHD cascade have sufficiently small scales to come into resonant interactions with positrons.

\subsection{Positron interactions with MHD wave cascades}

Our previous discussion indicates that the MHD cascades are truncated at scales several orders of magnitude larger than the Larmor radii of positrons produced by radioactive decay or as cosmic rays, except in the hot and warm ionized phases of the ISM. In these phases, Alfvén wave turbulence is expected to cascade nearly undamped down to scales close to the Larmor radius of $\mathrm{MeV}$ positrons. However, as we now argue, this does not necessarily mean that short-wavelength Alfvén waves will resonantly interact with $\mathrm{MeV}$ positrons. Indeed, magnetic fluctuations are probably highly anisotropic at small scales, in the sense that turbulent eddies are strongly elongated along the mean magnetic field, or, in mathematical terms, $k_{\perp} \gg k_{\|}$(Goldreich \& Sridhar 1995; Yan \& Lazarian 2004). Because of the important anisotropy of magnetic fluctuations, which increases towards smaller scales, scattering off Alfvén waves appears questionable. The elongated irregularities associated with anisotropic turbulence average out over a particle gyration (Chandran 2000). If some Alfvén waves are present at scales $\sim r_{\mathrm{L}}^{\prime}$, the scattering frequency is reduced by more than 20 orders of magnitude compared to the situation with isotropic turbulence (see, for instance, 
Yan \& Lazarian 2002). Slab turbulence gives the same order of estimates (Yan \& Lazarian 2004). In consequence, scattering off Alfvén wave turbulence should be extremely inefficient at confining positrons in the ionized phases of the ISM. In this view, the diffusion models for positron transport adopted in a series of recent papers by Parizot et al. (2005); Cheng et al. (2006); Jean et al. (2006), using diffusion coefficients derived from quasilinear theory, overestimate the confinement by plasma waves.

To circumvent this problem, Yan \& Lazarian (2004) reconsidered scattering off fast magnetosonic waves, emphasizing the isotropy of the fast wave cascade. As we saw earlier, in the hot and warm ionized phases, fast waves decay away at scales much larger than the Larmor radii of positrons from radioactivity, except possibly at quasi-parallel propagation. If propagation angles are not or only weakly randomized by wave-wave interactions or by chaotic divergence of magnetic field lines, quasi-parallel waves may survive down to much smaller scales. In that case, they may be involved either in gyroresonance or in Cherenkov resonance, also known as transit-time damping (TTD) resonance, with positrons. As explained at the end of Sect. 2.1, Cherenkov resonance occurs at propagation angles $\Theta_{\mathrm{T}}$ such that $\cos \Theta_{\mathrm{T}} \simeq V_{\mathrm{ms}} /(v \cos \alpha)$, independent of the wavelength. But we know that only those fast waves with $\Theta_{\mathrm{T}} \simeq 0$ have a chance to escape heavy damping. From this, we conclude that only positrons with pitch angles satisfying $\cos \alpha \simeq V_{\mathrm{ms}} / v \ll 1$ have a chance to experience TTD resonance. At precisely $\cos \alpha=V_{\mathrm{ms}} / v$, the TTD mechanism vanishes, but its rate rises rapidly as $\cos \alpha$ increases above $V_{\mathrm{ms}} / v$ (Schlickeiser \& Miller 1998). Ultimately, positrons produced by radioactive decay are unlikely to efficiently interact with MHD waves from a direct cascade generated at large scales. Such positron-wave interactions appear to be completely ruled out in the neutral phases of the ISM. In the ionized phases, they could potentially take place, but only under very restrictive conditions, including quasi-parallel fast waves $\left(\Theta_{\mathrm{T}} \simeq 0\right)$ and nearly perpendicular positron motion $\left(\alpha \simeq \arccos \left(V_{\mathrm{ms}} / v\right)\right)$.

Of course, an additional local injection of MHD waves at much smaller scales could participate in the confinement of positrons. Some aspects of this possibility will be discussed in Sect. 2.5.

\subsection{Wave injection through plasma instabilities}

As we saw in the previous subsection, MHD waves injected at large scales into an MHD cascade are generally unable to efficiently interact with positrons from radioactive decay. However, MHD waves can be injected into the ISM by a variety of fluid or kinetic instabilities, which involve changes over the whole or a fraction of the velocity distribution of some particle population. These waves can be injected at scales $\ll L_{\text {inj }}$, possibly directly into the collisionless regime. Each case requires a dedicated investigation of the wave damping process. The scale of the turbulence injection is a key parameter controlling the interaction between MHD waves and positrons. First, $L_{\text {inj }}$ controls the anisotropy of the Alfvén wave cascade at the scale of the Larmor radius of positrons. Second, it also controls the cutoff wavenumber and the propagation angle of the fast magnetosonic cascade (see Eq. (A.18)). In this subsection, we focus on some particular aspects of one type of kinetic instability.

One of the most widely studied kinetic instabilities is triggered by the streaming of cosmic rays in the ISM, with a bulk velocity larger than a few times the local Alfvén speed (Wentzel 1974; Skilling 1975). The streaming instability is expected to develop mainly in the intercloud medium. Cosmic-ray streaming compensates for the sink in the low-energy cosmicray population due to strong ionization losses inside molecular clouds. Low-energy cosmic rays scatter off their selfgenerated waves, and are, therefore, excluded from molecular clouds (Lerche 1967; Skilling \& Strong 1976; Cesarsky \& Volk 1978; Dogel \& Sharov 1985). This scenario was adapted to the transport of cosmic-ray electrons by Morfill (1982). The streaming instability and other kinds of kinetic instabilities recently received new attention in the context of cosmic-ray diffusion in anisotropic MHD turbulence (Farmer \& Goldreich 2004; Lazarian \& Beresnyak 2006).

Here, we restrict our discussion to the sole streaming instability. The waves are generated at scales $k_{\text {st }}^{-1}$ close to the gyroradii of low-energy cosmic rays, so that $k_{\text {st }}^{-1} \ll L_{\text {inj. }}$. If the waves generated by cosmic rays are to serve as scattering agents for low-energy positrons, then the Landau-synchrotron resonance condition has to be fulfilled by both species, i.e., by virtue of Eq. (3):

$p_{\mathrm{e}}=p_{\mathrm{p}} \frac{\left|\cos \alpha_{\mathrm{p}}\right|}{\left|\cos \alpha_{\mathrm{e}}\right|}$

Equation (19) implies that the wave-generating cosmic rays and the scattered positrons must have comparable momenta, unless the ratio of angular factors is very different from unity. Now, the wave-generating cosmic rays have typical momenta in the range $\left[p_{\min }, p_{\max }\right]$. Their maximum momentum is set by the condition that waves can indeed be amplified by cosmic-ray streaming, which leads to $p_{\max } \sim 100 \mathrm{MeV} / c$ (Skilling \& Strong 1976). Their minimum momentum is set by the highest possible frequency of MHD waves, exactly as for positrons, and is therefore given by $p_{\min } \simeq m_{\mathrm{p}} V_{\mathrm{A}}$ (see Eq. (7)). This means that, in the warm ionized phase, positrons with kinetic energies below $\simeq 2.5-10 \mathrm{keV}$ (depending on the exact ion density) can no longer resonate with Alfvén waves generated by cosmic-ray streaming (see Table 1). We conclude that this mechanism does not operate over a broad range of positron momenta and that it probably does little to confine positrons within the ionized phases of the ISM.

Higdon et al. (2009) proposed an alternative mechanism whereby positrons scatter off their own self-generated waves. The growth rate of such an instability is proportional to the density of resonant particles. The positron density depends strongly on their position with respect to the sources. Higdon et al. (2009) argued that whistler waves are heavily damped in the neutral phases of the ISM, but the streaming instability may also operate close to the sources (for instance close to supernova remnants; see Ptuskin et al. 2008) or above the Galactic disk where the ionization fraction can be close to unity. A complete estimation of this process deserves a detailed investigation and will be considered in a forthcoming paper.

\subsection{Positron transport in dissipated turbulence and re-acceleration}

In the solar wind, $\mathrm{MeV}$ positrons can also interact with perturbations that fall into the dissipative range of the turbulence, above the steepening observed at a fraction of the proton gyrofrequency at wavenumbers $k \gtrsim k_{\mathrm{A}}$. It has been proposed that low-frequency non-resonant magnetosonic waves can dominate the propagation of sub-MeV particles if magnetosonic waves are present in the solar wind (Toptygin 1985; Ragot 2006). Ragot (1999) suggested that non-resonant fast magnetosonic waves can produce efficient angular scattering through pitch angles 
$\alpha \rightarrow \pi / 2$ and thus govern the particle mean free-path at energies $\sim 1 \mathrm{MeV}$. This result could be applied to the stellospheres of massive stars (about a few parsecs) and to larger regions of the ISM (see discussion in Higdon et al. (2009), though in a different perspective).

The electric component of the waves induces a variation in particle energy. The average effect of such interactions leads to a stochastic energy gain, also known as second-order Fermi acceleration. Particle stochastic acceleration (also referred to as reacceleration) is important if the re-acceleration time is shorter than the energy loss time, i.e., if $t_{\text {stoch }} \lesssim t_{\text {loss }}$. The re-acceleration time is related to the momentum diffusion coefficient $D_{p}$ through $t_{\text {stoch }}=p^{2} / D_{p}$ (here, we consider only pitch-angle averaged quantities). It can also be expressed in terms of the particle angular diffusion frequency $v_{\mathrm{s}}$ as $t_{\text {stoch }} \simeq\left(c / V_{\mathrm{A}}\right)^{2} v_{\mathrm{s}}^{-1}$. Then the above timescale ordering is equivalent to

$v_{\mathrm{s}} \gtrsim\left(\frac{c}{V_{\mathrm{A}}}\right)^{2} \frac{1}{t_{\mathrm{loss}}}$.

Particles with energies $\lesssim 1 \mathrm{GeV}$ mostly suffer ionization and Coulomb losses. The energy loss rate depends on whether the medium is ionized or neutral. Ginzburg (1979) gives

$$
\frac{1}{t_{\mathrm{loss}}}=\frac{3}{4} \sigma_{\mathrm{T}} c n_{\mathrm{e}} \frac{73.6+\ln \left(\gamma / n_{\mathrm{e}, \mathrm{cm}}{ }^{-3}\right)}{\beta(\gamma-1)}
$$

in an ionized medium and

$$
\frac{1}{t_{\text {loss }}}=\frac{3}{4} \sigma_{\mathrm{T}} c n_{\mathrm{H}^{0}} \frac{20.5+\ln \left[(\gamma-1)\left(\gamma^{2}-1\right)\right]}{\beta(\gamma-1)}
$$

in a neutral medium, where $\sigma_{\mathrm{T}}$ is the Thomson cross-section, $\beta=v / c$ and, as before, $\gamma$ is the particle Lorentz factor, $n_{\mathrm{e}}$ the free-electron density and $n_{\mathrm{H}^{0}}$ the density of hydrogen atoms. The condition (20) translates into an upper limit on the mean free-path:

$$
\lambda \lesssim\left(3.4 \times 10^{-5} \mathrm{pc}\right)\left(\frac{B_{\mu \mathrm{G}}^{2}}{n_{\mathrm{e}, \mathrm{cm}^{-3}} n_{\mathrm{i}, \mathrm{cm}^{-3}}}\right) \frac{(\gamma-1) \beta^{2}}{\ln \left(\gamma / n_{\mathrm{e}, \mathrm{cm}^{-3}}\right)+73.6}
$$

in an ionized medium and

$$
\begin{aligned}
\lambda \lesssim & \left(3.4 \times 10^{-5} \mathrm{pc}\right)\left(\frac{B_{\mu \mathrm{G}}^{2}}{n_{\mathrm{H}^{0}, \mathrm{~cm}^{-3} n_{\mathrm{i}, \mathrm{cm}^{-3}}}}\right) \\
& \times \frac{(\gamma-1) \beta^{2}}{\ln \left[(\gamma-1)\left(\gamma^{2}-1\right)\right]+20.5}
\end{aligned}
$$

in a neutral medium. This implies that if, in a particular medium, the angular diffusion frequency is high enough, particles are reaccelerated. In that case, a solution can be obtained with the help of a diffusion-convection equation. This type of investigation is beyond the scope of the current paper and is postponed to a future work. However, the above discussion already calls some of the results derived by Higdon et al. (2009) into question, as these authors ignored re-acceleration in their analysis. What emerges from the above discussion is that re-acceleration can be important, especially in low-density ionized phases. More specifically, if we introduce the parameter values proposed by Higdon et al. (2009) for the Galactic interstellar bulge (see their Table 1), we find that positron re-acceleration is important in the very hot phase of the inner bulge, and probably in the hot phase of the middle and outer bulge.

\section{Effects of collisions with gas particles}

In this section, we examine the case when the trajectories of positrons in the ISM is only driven by their collisions with gas particles while they propagate along a steady state magnetic field line, without considering interactions with MHD waves, an aspect usually overlooked by the previous analysis. During these collisions, high-energy positrons not only lose energy, but they also undergo pitch angle scattering. The scattering of kinematic parameters of positrons reduces the maximum distance they can travel along a straight line in the ISM. In Sect. 3.1, we review and describe the interaction processes between positrons and interstellar matter. In Sect. 3.2, we describe the methods used to calculate positron propagation in this so-called "collisional regime", and in Sect. 3.3, we present the detailed results of our computations for $1 \mathrm{MeV}$ positrons. The main results obtained for positrons with initial kinetic energies ranging from $1 \mathrm{keV}$ to $10 \mathrm{MeV}$ in typical ISM phases and the case of propagation in a turbulent magnetic field are described in Sect. 3.4.

\subsection{Positron interactions with interstellar matter}

In the absence of collisions, positrons move along magnetic field lines in helical trajectories. When positrons interact with gas particles, they can either gain or lose energy in elastic and inelastic scattering or even annihilate with free or bound electrons. The energy and pitch angle variations resulting from the interaction depend on the energy of the incident positron and on the velocity and nature of the target particle.

The positrons that we are studying spend most of their lifetime travelling at high energy, with a kinetic energy greater than the thermal energy of target particles in the ISM. Consequently, we do not account for the propagation of positrons at thermal energy, but postpone the discussion of this case to Sect. 3.4.

Interactions of positrons with the ionized component of the interstellar gas and, at high energy ( $E \gtrsim 100 \mathrm{MeV})$, with the magnetic field (synchrotron radiation) and the interstellar radiation field (inverse Compton scattering) are generally considered as continuous processes. They are referred to as continuous energy-loss processes and are presented in Sect. 3.1.1. In a mostly neutral gas, positrons lose a larger fraction of their energy when they excite or ionize atoms or molecules. Such interactions, which result in a quantified variation of the positron energy, are described in Sect. 3.1.2.

\subsubsection{Continuous energy-loss processes}

Figure 1 shows the energy loss rates of positrons as functions of their energy in a fully ionized plasma with temperature $T=8000 \mathrm{~K}$. The energy loss rates by synchrotron radiation and inverse Compton scattering are derived from Blumenthal \& Gould (1970). They are proportional to the magnetic-field and photon energy densities, respectively. The energy loss rate by bremsstrahlung is calculated using the approximation presented in Ginzburg (1979). The energy loss rate due to Coulomb collisions (free-free) is calculated using the Bhabha cross-section as described in Asano et al. (2007).

In a fully ionized plasma, Coulomb collisions represent the dominant loss process for positrons with $E \lesssim 100 \mathrm{MeV}$. Since the energy loss rates by synchrotron radiation and inverse Compton scattering depend on the magnetic-field and photon energy densities, they vary with the location of positrons in our Galaxy. It is expected that inverse Compton losses become dominant in zones close to stellar clusters. However, we are interested in positrons 


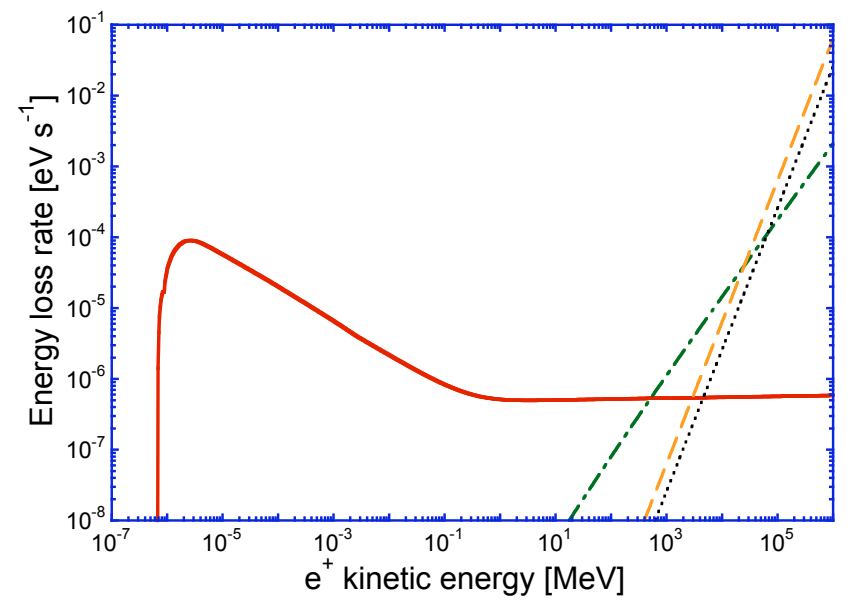

Fig. 1. Energy-loss rates of positrons in a $8000 \mathrm{~K}$ plasma with number densities $n_{\mathrm{e}}=n_{\mathrm{H}^{+}} \simeq 1 \mathrm{~cm}^{-3}$. The long-dashed line shows the energy-loss rate due to synchrotron radiation assuming $B_{0}=5 \mu \mathrm{G}$ and an average value of $\sin ^{2} \alpha=2 / 3$. The dotted line is the energy-loss rate by inverse Compton scattering assuming $U_{\mathrm{ph}}=0.26 \mathrm{eV} \mathrm{cm}^{-3}$, which corresponds to the cosmic microwave background energy density. The dot-dashed line shows the energy-loss rate by bremsstrahlung emission due to collisions with charged particles. The solid line corresponds to the positron energy-loss rate due to Coulomb interactions between positrons and electrons.

with kinetic energy $\lesssim 10 \mathrm{MeV}$ and, therefore, we may neglect synchrotron losses as long as $B \lesssim 1 \mathrm{mG}$.

The average scattering angle induced by Coulomb collisions occurring over a time interval $\delta t$ is estimated through the relation

$\sin \bar{\theta}=\sqrt{\frac{\mathrm{d}\left\langle\sin ^{2} \theta\right\rangle}{\mathrm{d} t} \delta t}$,

where $d\left\langle\sin ^{2} \theta\right\rangle / d t$ is the rate of variation of the average $\sin ^{2} \theta$ and $\theta$ is the positron scattering angle in the laboratory frame. The rates of variation of the average scattering angle are evaluated by integrating the Bhabha $\left(\mathrm{e}^{+} \mathrm{e}^{-}\right.$collision) and Rutherford $\left(\mathrm{e}^{+} \mathrm{p}\right.$ collision) differential cross sections (see Appendix in Asano et al. 2007), over the scattering angles as

$\frac{\mathrm{d}\left\langle\sin ^{2} \theta\right\rangle}{\mathrm{d} t}=v n \int_{\theta_{\min }^{\star}}^{\pi} \sin ^{2} \theta \frac{\mathrm{d} \sigma^{\star}}{\mathrm{d} \cos \theta^{\star}} \sin \theta^{\star} \mathrm{d} \theta^{\star}$,

where $n$ is the number density of target particles, $\theta^{\star}$ is the scattering angle in the center-of-mass frame, $\frac{\mathrm{d} \sigma^{\star}}{\operatorname{dcos} \theta^{\star}}$ is the differential cross section of the interaction and $\theta_{\text {min }}^{\star}$ is the minimum scattering angle (see Eq. (B7) in Dermer 1985).

While the energy loss rate from $\mathrm{e}^{+} \mathrm{p}$ collisions is negligible compared to that from $\mathrm{e}^{+} \mathrm{e}^{-}$collisions, their deviation rates are equivalent. We checked that the resulting deviation rates are in agreement with those derived at low energy using the formalism of Huba (2006).

\subsubsection{Annihilation and interactions with atoms and molecules}

In a neutral medium, high-energy positrons lose energy mainly by ionizing and exciting atoms and molecules, or they annihilate directly with bound electrons, the elastic scattering process being negligible at these energies (see Charlton \& Humberston 2000 and Wallyn et al. 1994). Such interactions occur at random while positrons propagate in the ISM. Therefore,

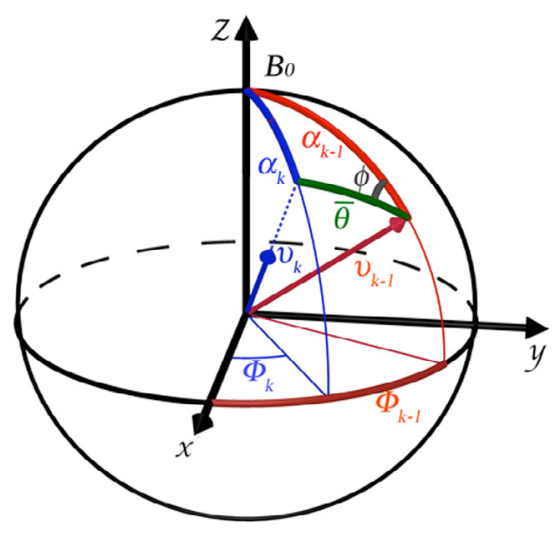

Fig. 2. Kinematic parameters of the scattering in the laboratory frame. The magnetic field $\boldsymbol{B}_{0}$ lies along the $z$ axis. $\alpha_{k-1}$ is the pitch angle between the positron velocity $\boldsymbol{v}_{k-1}$ and $\boldsymbol{B}_{0}$ before the interaction. $\alpha_{k}$ is the pitch angle after the interaction, when the positron velocity is $\boldsymbol{v}_{k}$. $\Phi_{k-1}$ and $\Phi_{k}$ are the phases of incident positrons before and after the interaction, respectively. $\bar{\theta}$ is the scattering angle of positrons and $\phi$ is the azimuthal scattering angle.

we will determine the kind of interaction and calculate the variation of the kinematic parameters with a Monte-Carlo method that incorporates the corresponding cross sections.

The ionization and excitation cross sections as well as the differential cross sections as functions of the energy lost by positrons in ionizing collisions were calculated by Gryziński (1965a,b,c). The cross section of annihilation in flight of positrons with bound electrons is equal to that of annihilation with free electrons, since the binding energy of electrons is negligible with respect to the kinetic energy of the positrons under study. We use the cross section of annihilation with free electrons presented in Guessoum et al. (2005, and references therein).

In the Monte-Carlo simulations, the energy lost by a positron when it ionizes an atom/molecule is chosen randomly according to its differential cross section. The energy lost by a positron when it excites an atom/molecule is derived from the energies of the atomic levels involved in the interaction. Once the energy lost by ionization or excitation is known, the scattering angle of the positron is calculated with the kinematics of the interaction, assuming that the atoms/molecules stay at rest and assuming azimuthal symmetry.

\subsection{Simulations of the collisional transport}

Our Monte-Carlo simulations are based on the methods presented in Bussard et al. (1979) and Guessoum et al. (2005). However, we add the calculation of the trajectories of positrons while they propagate along magnetic field lines and include additional steps to account for the scattering of positrons by collisions.

At the initial time $(k=0)$, positrons are located at $(x, y, z, t)=(0,0,0,0)$ in a frame such that the magnetic field $\boldsymbol{B}_{\mathbf{0}}$ is directed along the $z$ axis. The initial kinetic energy of positrons is $E_{0}$. The direction of their initial velocity $\boldsymbol{v}_{0}$, which is defined by their initial pitch angle $\alpha_{0}$ and their initial phase $\Phi_{0}$ (see Fig. 2), is chosen randomly according to an isotropic velocity distribution in the entire space.

We proceed through successive iterations. At step $k$, the resulting pitch angle $\left(\alpha_{k}\right)$ and phase $\left(\Phi_{k}\right)$ are given by

$\cos \alpha_{k}=\cos \alpha_{k-1} \cos \bar{\theta}+\sin \alpha_{k-1} \sin \bar{\theta} \cos \phi$, 
$\cos \left(\Phi_{k}-\Phi_{k-1}\right)=\frac{\cos \bar{\theta}-\cos \alpha_{k-1} \cos \alpha_{k}}{\sin \alpha_{k-1} \sin \alpha_{k}}$

where the azimuthal scattering angle $\phi$ is a random number uniformly distributed between 0 and $2 \pi$. The kinetic energy $E_{k}$ after collision with an atom/molecule or annihilation with an electron is given by Bussard et al. (1979) :

$R=\exp \left[-\int_{E_{k-1}}^{E_{k}} \frac{v \sum_{i, j} n_{j} \sigma_{i, j}(E)}{\mathrm{d} E / \mathrm{d} t} \mathrm{~d} E\right]$,

where $R$ is a random number uniformly distributed between 0 and $1, \sigma_{i, j}$ is the cross section of process $i$ (see Sect. 3.1.2) with target $j$ and $n_{j}$ is the number density of target $j$. The kind of interaction is chosen randomly according to its probability

$P_{i, j}\left(E_{k}\right)=\frac{n_{j} \sigma_{i, j}\left(E_{k}\right)}{\sum_{i, j} n_{j} \sigma_{i, j}\left(E_{k}\right)}$,

The energy lost and the scattering angle induced by collisions are derived using the methods presented in Sect. 3.1.2.

The increment in time between two iterations, $\delta t$, is equal to a scattering time $\delta t_{\mathrm{s}}$ defined as

$\delta t_{\mathrm{s}}=\frac{\epsilon}{\mathrm{d}\left\langle\sin ^{2} \theta\right\rangle / \mathrm{d} t}$,

with $\mathrm{d}\left\langle\sin ^{2} \theta\right\rangle / \mathrm{d} t$ the rate of variation of the average $\sin ^{2} \theta$ (see Eq. (26)) and $\epsilon$ a prescribed number $\ll 1$. During $\delta t_{\mathrm{s}}$, positrons lose an amount of energy $\mathrm{d} E / \mathrm{d} t \times \delta t_{\mathrm{s}}$ via continuous energy-loss processes, and, they are slightly scattered by an angle $\bar{\theta}$ obtained from Eq. (25). If the time interval between two successive collisions with neutrals is shorter than the scattering time $\delta t_{\mathrm{s}}$ (e.g., in a weakly ionized medium), then the increment in time $\delta t$ is replaced by the time interval between two collisions.

Between two steps and/or two interactions, positrons propagate in a regular helical trajectory along magnetic field lines with a gyroradius $r_{\mathrm{L}}^{\prime} \sin \alpha_{k-1}$ (defined in Sect. 2.1). The positron position after each step is therefore calculated analytically.

The above procedure is repeated until positrons annihilate or their kinetic energy falls below $100 \mathrm{eV}$, since the distance traveled below this energy becomes negligible. To estimate the spatial distribution of positrons and their lifetime, we read out the positron location and slowing-down time at the end of the track. To save CPU time while obtaining sufficiently accurate results, Monte-Carlo simulations are performed with a number of positrons ranging from 5000 to 20000 .

To validate our code, we performed several tests for positrons with $E_{0}<10 \mathrm{MeV}$ released in different media and we compared the results of our simulations with previous work. The fractions of positrons annihilating in flight with free or bound electrons are in agreement with the results presented by Beacom \& Yüksel (2006) and Sizun et al. (2006). At low energy $\left(E_{0}<1 \mathrm{keV}\right)$, the fractions of positronium formed in flight by charge exchange with atoms and molecules in different media are identical to those obtained by Guessoum et al. (2005). Once positrons are thermalized, their propagation behaves as classical diffusion. The distance they travel then is negligible compared to the distance traveled in the slowing-down regime, except in the hot medium (see Sect. 3.4).

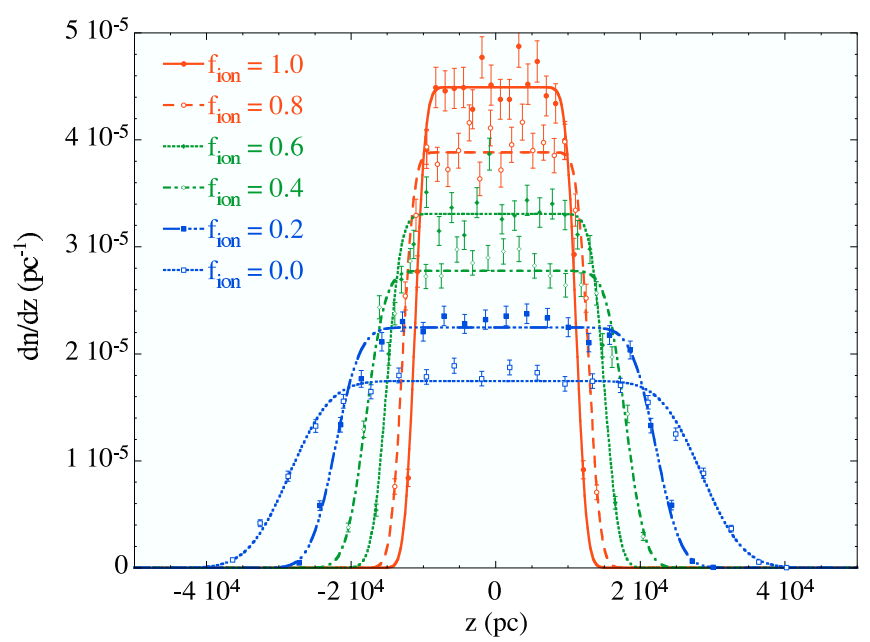

Fig. 3. Spatial distributions of positrons along field lines at the end of their slowing-down period in a warm medium $(T=8000 \mathrm{~K}$ and $n_{\mathrm{H}}=1 \mathrm{~cm}^{-3}$ ), for several ionization fractions, $f_{\text {ion }}$. The distributions are normalized to unity and the error bars are estimated according to Poisson (counting) statistics.

\subsection{Results of the simulations for $1 \mathrm{MeV}$ positrons}

This section presents, as an illustrative example, the detailed results of simulations obtained for positrons with initial kinetic energy $E_{0}=1 \mathrm{MeV}$. For this first set of simulations, we chose the warm medium, whose temperature is intermediate between those of the cold and hot media. The warm medium is also the most appropriate medium to study the impact of the ionization fraction, which covers the whole possible range between 0 and 1 . We assumed the magnetic field to be uniform, with a strength $B_{0}=5 \mu \mathrm{G}$.

Since the energy loss rates and the frequency of inelastic interactions are proportional to the target density, for given abundances and a given ionization fraction, the distance traveled by positrons scales as the inverse of the total density. Therefore, we performed simulations for $n_{\mathrm{H}}=1 \mathrm{~cm}^{-3}$, with $n_{\mathrm{H}}=n_{\mathrm{H}^{0}}+n_{\mathrm{H}^{+}}+2 n_{\mathrm{H}_{2}}$ the total number density of hydrogen nuclei. Preliminary tests of our simulations indicate that the presence of ionized or neutral helium with an abundance ratio $\mathrm{He} / \mathrm{H} \approx 0.1$ does not strongly affect positron propagation (differences less than 10\%). We also neglected molecular hydrogen, except in the MM where $\mathrm{H}_{2}$ molecules are the dominant species.

Figure 3 shows the spatial distributions of positrons along the magnetic field direction $(z)$, as extracted from our simulations, once they reach $E=100 \mathrm{eV}$. Positrons are initially "injected" at $(x, y, z)=(0,0,0)$ with a pitch angle chosen randomly according to an isotropic velocity distribution in the entire space. The temperature is $8000 \mathrm{~K}$ and the ionization fraction ranges from 0 to 1 . The field-aligned distributions are nearly uniform out to the maximum distance traveled along field lines, $d_{\max }$ (obtained when the pitch angle is and remains equal to 0 ). This means that the pitch angle does not change significantly over most of the slowing-down period of positrons. If the pitch angle remained strictly constant, the distance traveled along field lines would be equal to $d_{\max } \times \cos \alpha_{0}$. Since positrons are initially emitted isotropically, $\cos \alpha_{0}$ is uniformly distributed between -1 and 1 , so that the field-aligned distributions of positrons at the end of their slowing-down period would be uniform. In reality, the slight pitch angle scattering induced by collisions produces a slight scattering of their final field-aligned positions and, therefore, smoothes out the edge of the otherwise uniform 


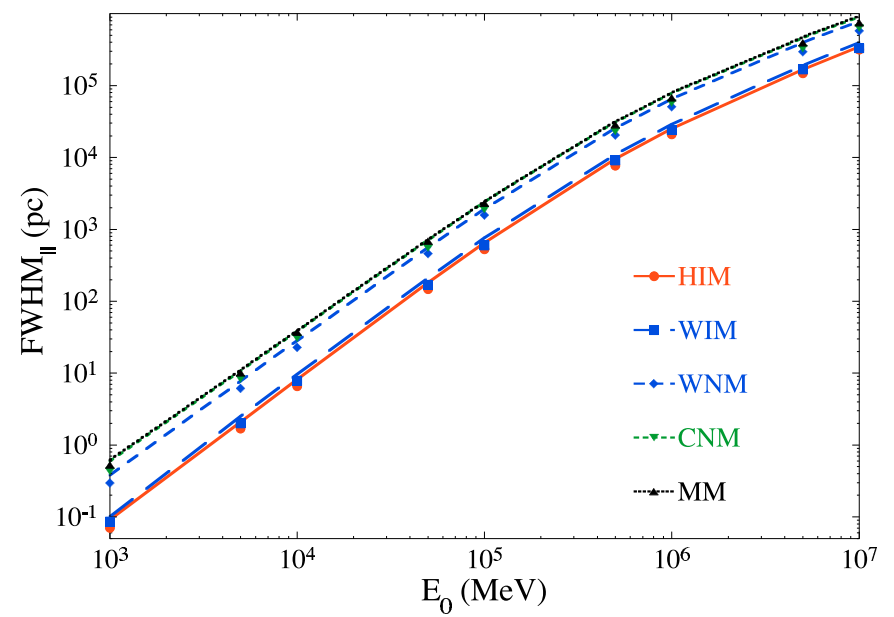

Fig. 4. FWHM of the field-aligned distributions of at the end of their slowing-down period, as functions of their initial kinetic energy. The hydrogen density is arbitrarily set to $n_{\mathrm{H}}=1 \mathrm{~cm}^{-3}$ in each ISM phase. The lines show the distance $2 d_{\max }$ (see text for details) while points show $\mathrm{FWHM}_{/ /}$of the distribution as obtained by the simulations.

distributions. The maximum distance $d_{\max }$ is obtained by integrating over energy the ratio of the positron velocity to the energy loss rate. In the present case, the energy loss rate is the sum of the contributions from Coulomb collisions (see Sect. 3.1.1) and inelastic interactions (ionization and excitation) with atoms and molecules. The latter contributions are evaluated using the Bethe-Bloch formula (Ginzburg 1979).

The extent of the spatial distributions in the directions perpendicular to the magnetic field is a few times the Larmor radius, i.e., negligible with respect to the extent of the field-aligned distributions. We characterize the extent of the field-aligned distributions presented in Fig. 3 by their full width at half maximum $\left(\mathrm{FWHM}_{/ /}\right)$. Its uncertainty is calculated with a bootstrap method taking into account the uncertainty in the number of positrons (Poisson statistics) in each spatial bin.

The extent of the field-aligned distributions increases naturally with decreasing ionization fraction (see Fig. 3), since the energy losses through Coulomb collisions dominate over the losses due to inelastic interactions (ionization and excitation) with neutrals. Depending on the ionization fraction, the half $\mathrm{FWHM}_{/ /}$is $15 \%$ to $30 \%$ lower than $d_{\max }$.

\subsection{Transport of positrons in the different ISM phases}

This section summarizes the results of the simulations for positrons with initial kinetic energies ranging from $1 \mathrm{keV}$ to $10 \mathrm{MeV}$, in the different ISM phases.

Figure 4 shows the extent $\left(\mathrm{FWHM}_{/ /}\right)$of the field-aligned distribution of positrons at the end of their slowing-down time (i.e., when they reach $100 \mathrm{eV}$ ), as a function of their initial kinetic energy in the different ISM phases. As in Sect. 3.3, the hydrogen density is arbitrarily set to $n_{\mathrm{H}}=1 \mathrm{~cm}^{-3}$, and the ionization fraction is $0,0.001,0.1,0.9$ and 1 in the MM, CM, WNM, WIM and HIM, respectively. The extents presented in Fig. 4 are compared to the extents that the distributions would have if the positron pitch angles remained constant, namely, $2 d_{\max }$. In each ISM phase and for any given initial energy, $\mathrm{FWHM}_{/ /}$is similar to, and always slightly less than, $2 d_{\max }$, as expected from the effect of pitch angle scattering.
The above $\mathrm{FWHM}_{/ /}$extents are given assuming a uniform magnetic field in the $z$ direction. However, field lines in the ISM are perturbed by turbulent motions. As a result, realistic magnetic fields in the ISM consist of a mean ("regular") component plus a turbulent component, leading to chaotic field lines. The distances traveled by positrons along the uniform field (i.e., along the $z$ axis) are shorter than the total distances traveled along the actual chaotic field lines.

We estimate the effects of the turbulent magnetic field on the distances traveled by positrons by adding a turbulent component to the uniform field. This turbulent field is modelled using the plane wave approximation method presented by Giacalone \& Jokipii (1994). We assume a ratio $\delta B / B_{0} \simeq 1$. The fluctuations follow a Kolmogorov spectrum and have a maximum turbulent scale $\lambda_{\max } \simeq 10-100 \mathrm{pc}$ in the hot and warm phases and $\lambda_{\max } \simeq 1-10 \mathrm{pc}$ in the cold neutral and molecular phases. These scale lengths correspond to the typical sizes of the respective phases. Since the Larmor radii of positrons with kinetic energies in the considered range are extremely small compared to the turbulent scale lengths, positrons simply propagate along the turbulent field lines. Under these conditions, the actual coordinates of positrons at the end of their slowing-down period are obtained by carrying their coordinates along the uniform field (as calculated in Sect. 3.3) over to the curvilinear frame of the turbulent field lines. These coordinates are calculated by MonteCarlo simulations, using a large number of randomly chosen turbulent configurations for a given $\lambda_{\max }$. Figure 5 shows examples of the positions of positrons at the end of the slowing-down time, calculated with this method in a WIM with $n_{\mathrm{H}}=0.2 \mathrm{~cm}^{-3}$ and $f_{\text {ion }}=0.6$.

Adding such a turbulent magnetic field in the simulations reduces the FWHM of the positron field-aligned distributions by a factor $\simeq 0.75$ and broadens their transverse (i.e., perpendicular to $\boldsymbol{B}_{\mathbf{0}}$ ) distributions due to the chaotic behavior of field lines. At low energy, when the distance traveled by positrons is shorter than the maximum scale of the turbulence, $\lambda_{\max }$ (e.g., Fig. 5a), positrons have a quasi-uniform distribution along any field line that can be considered, at this scale, as straight and randomly tilted with respect to $\boldsymbol{B}_{\mathbf{0}}$. In this case, the spatial distribution of positrons is nearly spherically symmetric. At intermediate energy (e.g., Fig. 5b), positrons have a more collimated distribution with a transverse dispersion that increases with distance from the source. At high energy, when the distance traveled by positrons is large compared to $\lambda_{\max }$ (e.g., Fig. $5 \mathrm{c}$ ), the positron distribution is highly collimated along $\boldsymbol{B}_{\mathbf{0}}$.

The shape of the transverse distribution ${ }^{3}$ is strongly peaked, as illustrated in Fig. 6, for positrons with $E_{0}=1 \mathrm{MeV}$ propagating in a WIM. The transverse distribution is close to a decaying exponential function. Its extent increases with $\lambda_{\max }$, as expected since a larger scale length leads to larger transverse excursions of field lines away from the uniform field. In contrast, the extent of the field-aligned distribution does not change with $\lambda_{\max }$, because, on average, positrons propagate along the uniform magnetic field.

Due to the cusped shape of the transverse distribution, $\mathrm{FWHM}_{\perp}$ is not necessarily a very meaningful quantity, as the fraction of positrons inside $\mathrm{FWHM}_{\perp}$ is low. Moreover, this fraction changes significantly with $\lambda_{\max }$ (e.g., from $\simeq 1 \%$ to $\simeq 9 \%$ for the cases presented in Fig. 6) and with $E_{0}$ (e.g., from $\simeq 1 \%$ to $\simeq 30 \%$ for $E_{0}$ decreasing from $10 \mathrm{MeV}$ to $1 \mathrm{keV}$ ). In contrast,

\footnotetext{
3 The transverse distribution is $\frac{\mathrm{d} n}{\mathrm{~d} S}$ with $\mathrm{d} S=2 \pi r \mathrm{~d} r$ and $r$ the radius in the $x y$ plane.
} 

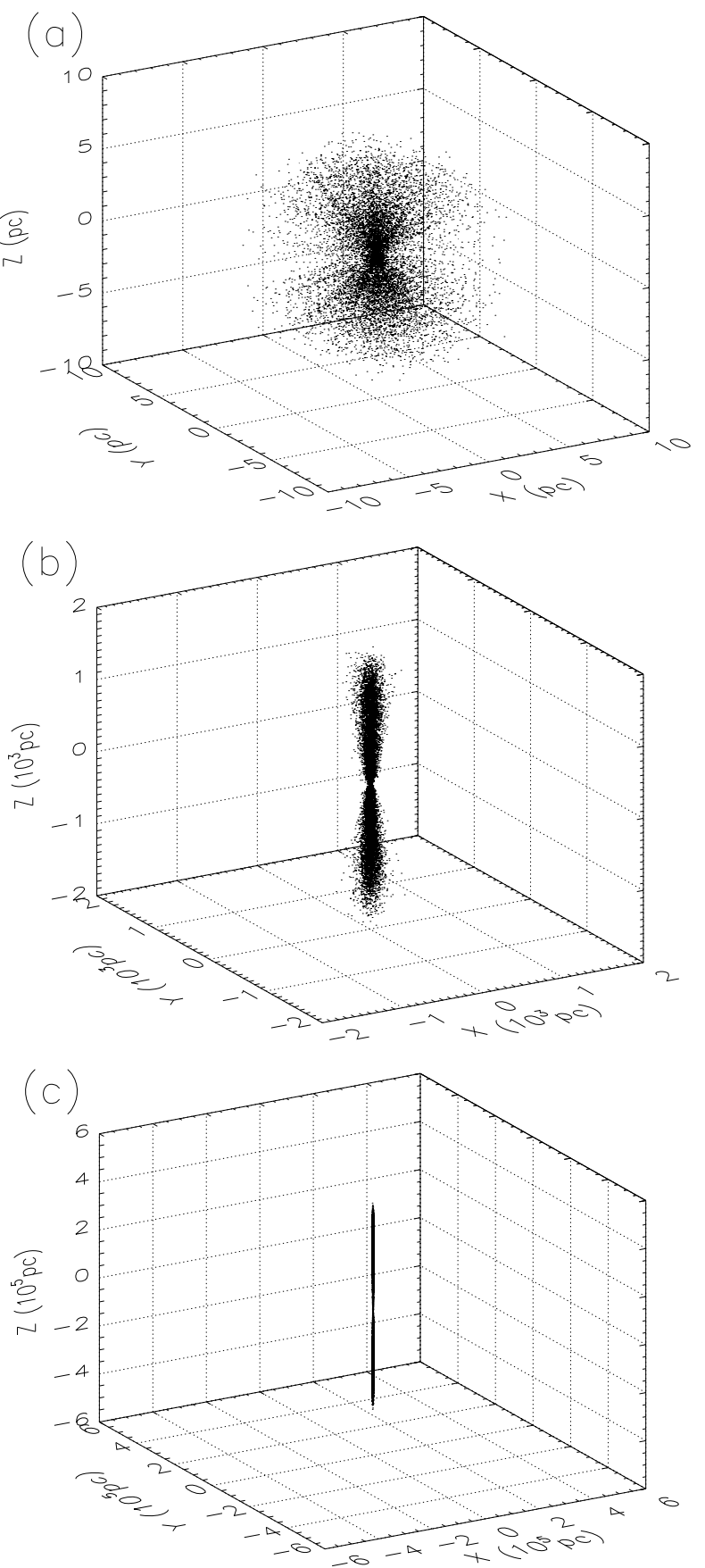

Fig. 5. Positions of positrons at the end of their slowing-down time in a WIM taking into account collisional transport in a turbulent magnetic field $\left(\delta B / B_{0}=1\right.$ and $\left.\lambda_{\max }=10 \mathrm{pc}\right)$. The uniform magnetic field lies along the $\mathrm{z}$ axis. Positrons are injected at the origin of the frame with initial kinetic energies of a) $5 \mathrm{keV}$, b) $100 \mathrm{keV}$ and c) $5 \mathrm{MeV}$.

the fraction of positrons inside the FWHM of the field-aligned distribution ranges from $\sim 80 \%$ to $\sim 95 \%$.

Consequently, instead of characterizing the spatial extents of the positron distribution by its FWHM, we will use the fieldaligned length $\left(2 z_{90}\right)$ and the diameter $\left(2 r_{90}\right)$ that contain $90 \%$ of the positrons. Figure 7 shows the extents of the spatial distribution as functions of the initial kinetic energy of positrons. The physical conditions (density, ionization fraction, temperature,...) of the different ISM phases correspond to the extreme values presented in Table 1 . The minimum and maximum spatial extents are obtained with the maximum and minimum densities

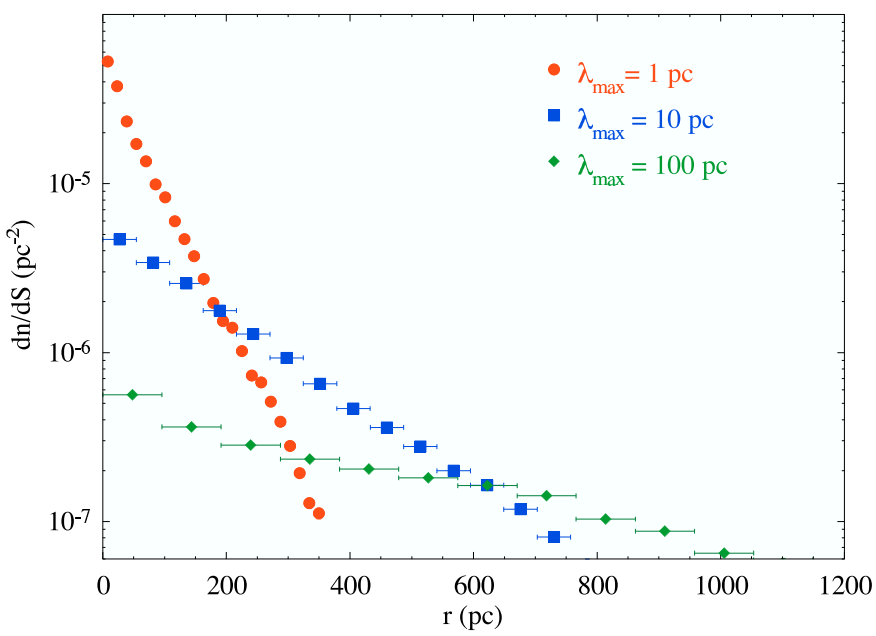

Fig. 6. Transverse distributions of positrons at the end of their slowingdown period in a WIM, calculated for maximum turbulent scales $\lambda_{\max }=$ 10,100 and 1000 pc. The initial kinetic energy of positrons is $E_{0}=$ $1 \mathrm{MeV}$. The distributions are normalized to unity.
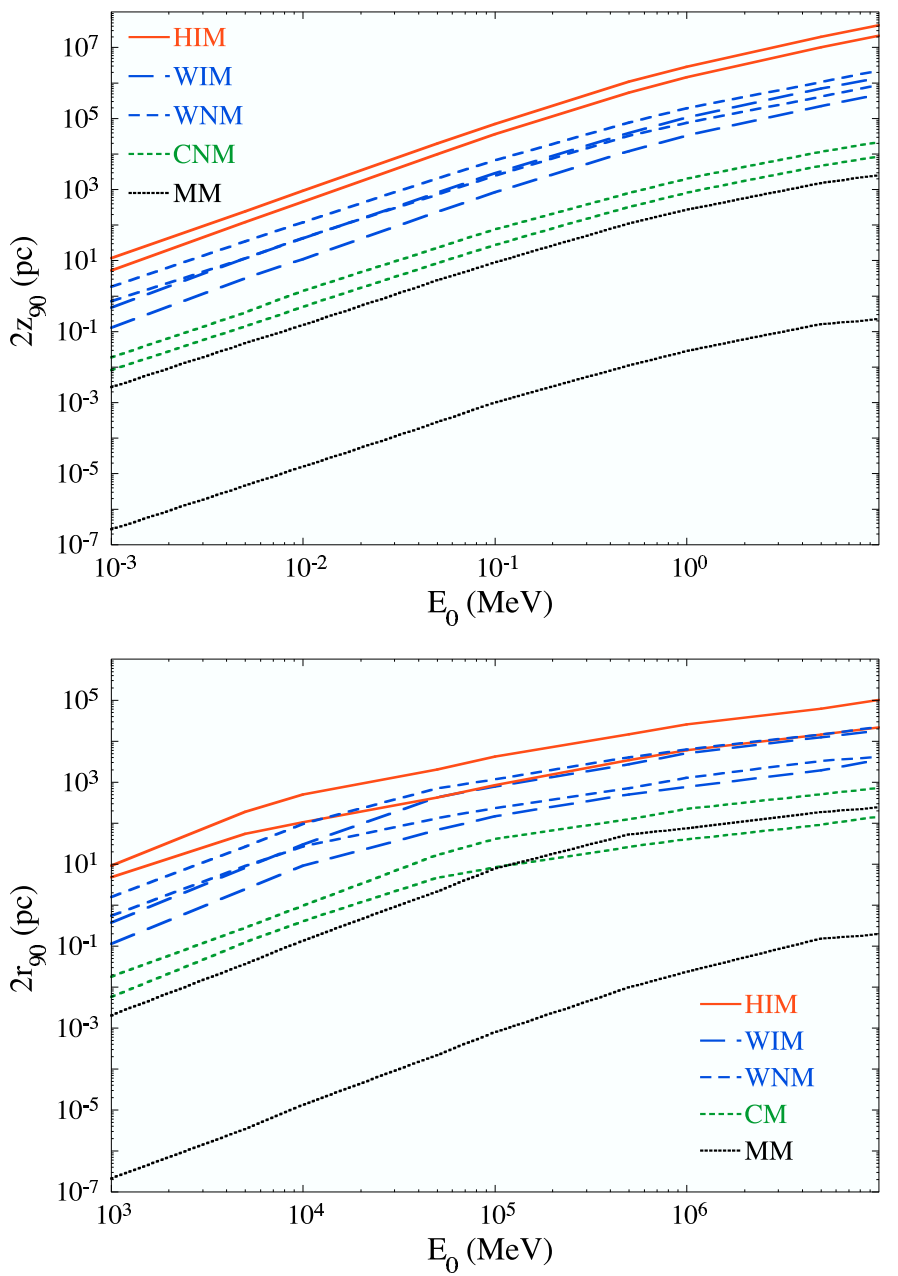

Fig. 7. Minimum and maximum extents of the spatial distributions of positrons reaching $100 \mathrm{eV}$, along (top) and perpendicular (bottom) to the uniform magnetic field, taking into account the turbulent behavior of the field lines as well as realistic values for the density in each ISM phase.

and ionization fractions listed in Table 1 and with the minimum and maximum $\lambda_{\max }$, respectively. 
The ratio $r_{90} / z_{90}$ increases up to $\sim 0.8$ with decreasing energy, confirming that the positron distribution is more isotropic at low energy. A spherically symmetric distribution would have produced a ratio $r_{90} / z_{90}=1.2$.

The results displayed in Fig. 7 show that $\mathrm{MeV}$ positrons travel long distances before reaching $100 \mathrm{eV}$. Positrons with $E_{0} \lesssim 30 \mathrm{keV}$ generally cannot escape molecular clouds (assuming the smallest size of molecular clouds is $\sim 1 \mathrm{pc}$ ). On the other hand, positrons with $E_{0} \gtrsim 7 \mathrm{keV}$ generally escape the hot medium (assuming the typical size of hot regions is $\sim 200 \mathrm{pc}$ ).

So far, we have discussed the distance traveled by positrons over their slowing-down period, which ends when their kinetic energy falls below $100 \mathrm{eV}$. We now consider the distance they travel before annihilating with electrons. In the neutral phases, low-energy positrons $(E<100 \mathrm{eV})$ annihilate mostly by forming positroniums through charge exchange processes with atoms or molecules. The distance they travel before annihilating is negligible compared to the distance traveled at high energy (see Table 4 of Jean et al. 2006). The situation is different in the ionized phases. There, positrons quickly thermalize and then annihilate with electrons (via radiative recombination or direct annihilation). Since the time scale of annihilation $\left(\tau_{\mathrm{ann}}\right)$ is inversely proportional to the electron density, positrons in low-density media may travel a long distance before annihilating. The rms distance traveled by thermalized positrons along a uniform magnetic field is

$z_{\mathrm{th}}=\sqrt{2 D_{\|} \tau_{\mathrm{ann}}}$,

with the diffusion coefficient $D_{\|}=\lambda_{\mathrm{e}} v_{\mathrm{e}} / 3$. Here, $v_{\mathrm{e}}$ is the positron rms velocity, $\lambda_{\mathrm{e}}$ is the positron collisional mean freepath,

$\lambda_{\mathrm{e}}=\left(2.5 \times 10^{13} \mathrm{~cm}\right) \frac{T_{\mathrm{e}, \mathrm{eV}}^{2}}{n_{\mathrm{e}, \mathrm{cm}^{-3}} \Lambda}$,

and values of $\tau_{\text {ann }}$ are derived from Table 3 of Guessoum et al. (2005).

The rms distance $z_{\text {th }}$ characterizes the width of the Gaussian field-aligned distribution of thermalized positrons at the time of annihilation, when they are initially injected isotropically at $z$ $=0$. Taking into account the turbulent magnetic field, the 3D spatial distribution of the sites where positrons annihilate is calculated by carrying their coordinates along the uniform field over to the curvilinear frame of the turbulent field lines. We then derive the field-aligned and transverse extents of this distribution ( $2 z_{\text {th, } 90}$ and $2 r_{\text {th }, 90}$, respectively), defined as the field-aligned length and the diameter that contain $90 \%$ of the annihilating positrons. In the WIM, $z_{\text {th }, 90}<0.015$ pc, i.e., $\ll z_{90}$. In the HIM, $z_{\text {th }, 90} \sim 300$ and $\sim 600 \mathrm{pc}$, for $n_{\mathrm{e}}=0.01$ and $0.005 \mathrm{~cm}^{-3}$, respectively. These values are $>z_{90}$ for positrons with $E_{0} \lesssim 10 \mathrm{keV}$ (see. Fig. 7). Furthermore, $z_{\text {th }, 90}$ in the HIM is larger than the typical size of hot regions, confirming the estimates of Jean et al. (2006) that positrons do not annihilate in the HIM.

Combining these results with the half extents $\left(z_{90}\right.$ and $\left.r_{90}\right)$ presented in Fig. 7, we calculate the actual full half-extents of the spatial distribution of annihilating positrons, as functions of their initial kinetic energy:

$z_{\mathrm{ann}, 90}=\sqrt{z_{90}^{2}+z_{\mathrm{th}, 90}^{2}}$

and

$r_{\mathrm{ann}, 90}=\sqrt{r_{90}^{2}+r_{\mathrm{th}, 90}^{2}}$.
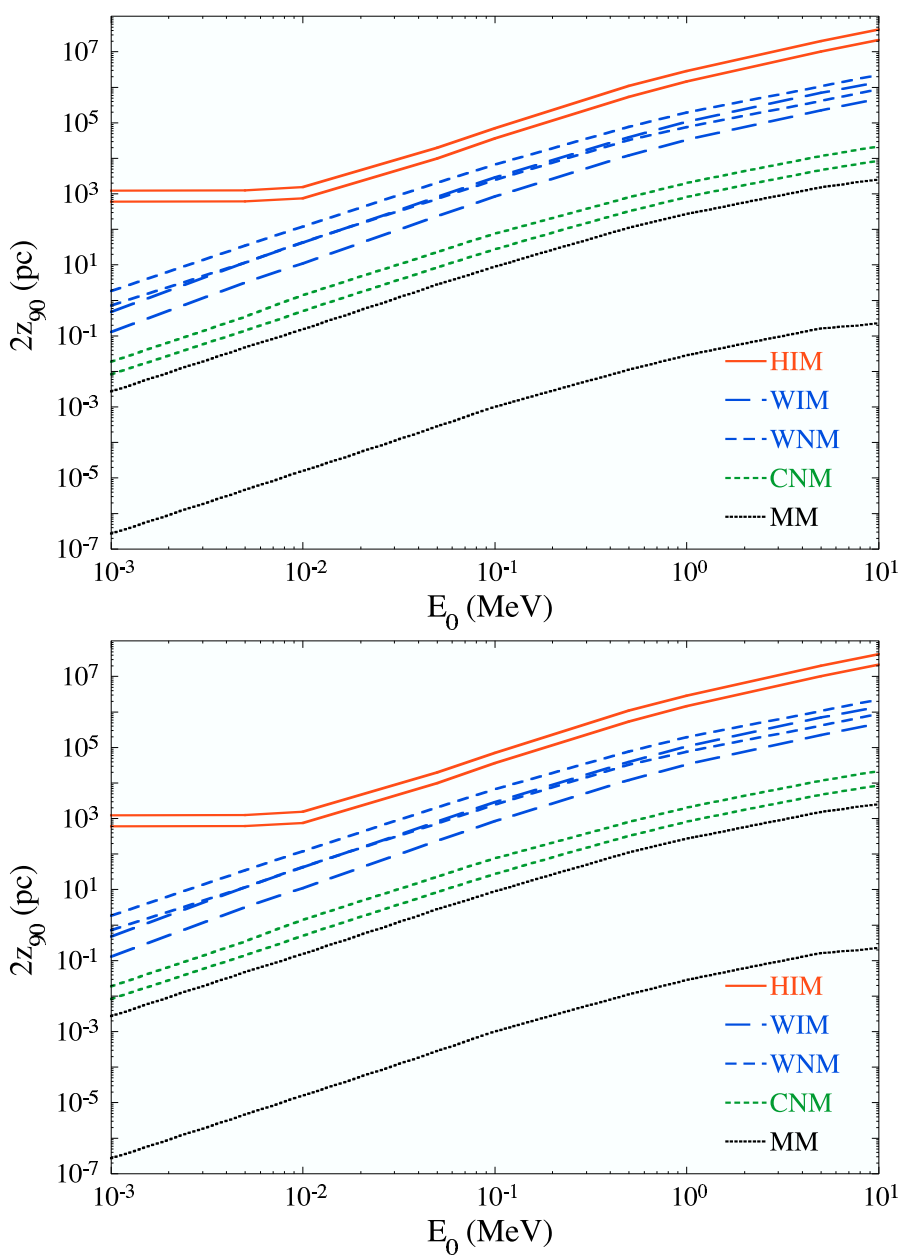

Fig. 8. Minimum and maximum extents of the spatial distributions of annihilating positrons, along (top) and perpendicular (bottom) to the uniform magnetic field, taking into account the turbulent behavior of the field lines as well as realistic values for the density in each ISM phase.

Figure 8 presents these extents in the different phases of the ISM. They are similar to the results presented in Fig. 7, except in the HIM where the lifetime of thermalized positrons is so long that they have time to travel a non-negligible distance before they annihilate.

\section{Advection with large-scale fluid motions}

As discussed in Sect. 2, low-energy positrons are at least loosely coupled to the thermal plasma, regardless of their production channel ( $\beta^{+}$decay or cosmic-ray interactions). More specifically, positrons are tied to magnetic field lines and the latter are frozenin in the plasma. If the plasma is subject to ordered or stochastic large-scale motions, positrons will be dragged along with the plasma and the field lines.

The Galactic center region is the site of intense massive star formation, as recently confirmed by long-exposure Chandra observations (Muno et al. 2006); it harbours within the innermost $30 \mathrm{pc}$ three bright and young star clusters: the Arches, the Quintuplet and the central Galactic cluster close to Sagittarius A*. Most massive stars are grouped into clusters and/or associations of up to several hundreds. The powerful winds from these stars together with their terminal supernova 
explosions act collectively to produce hot, low-density superbubbles (McCray \& Kafatos 1987; Parizot et al. 2004b). These superbubbles have a profound impact on the structure of the ambient ISM. Some of them are even powerful enough to break through the Galactic disk and produce chimneys, thereby venting their energy into the halo (McClure-Griffiths et al. 2006, and references therein).

Another possibility to drive strong winds in the Galaxy is again related to the streaming instability triggered by cosmic rays escaping the Galactic disk into the halo. This phenomenon may be rather effective at distances $\sim 1 \mathrm{kpc}$ from the Galactic center, at the transition between the diffusion and advection zones (Ptuskin et al. 1997). In reality, the transition could well be closer to the Galactic center. Recent HESS observations tend to support the idea that the cosmic-ray energy density around the Galactic center is higher than in the solar system (Aharonian et al. 2006).

The two next subsections examine some of these aspects in more detail.

\subsection{Massive-star forming regions: small-scale advection}

Positrons produced by radioactive decay in massive stars are typically injected into the hot interior cavity of a superbubble. In this hot medium, most positron transport takes place during the slowing-down period, a phase during which positrons are subject to collisions with gas particles.

In addition, positrons are advected with the motion of the plasma filling the superbubble. Because of the presence of strong stellar winds and supernova shocks inside the superbubble and because of their interactions with each other as well as with local density inhomogeneities, the hot cavity is undoubtedly highly turbulent (Bykov 2001, and references therein). According to Bykov (2001)'s model of inhomogeneous turbulence in superbubbles, positrons, which follow field lines, are subject to the same chaotic transport, characterized by a diffusion coefficient $D=v_{\text {rms }} L$. With a turbulent velocity in the range $v_{\text {rms }} \sim(100-1000) \mathrm{km} \mathrm{s}^{-1}$ and a mean scale of turbulent eddies in the range $L \sim(1-10) \mathrm{pc}$, corresponding to the typical distance between two stars (and two shocks), we find $D \sim\left(3 \times 10^{25}-3 \times 10^{27}\right) \mathrm{cm}^{2} \mathrm{~s}^{-1}$. It then follows that over their lifetime $\left(\tau_{\text {life }} \sim\left(10^{5} \mathrm{yr}\right) / n_{\mathrm{H}, \mathrm{cm}^{-3}}\right.$, with $n_{\mathrm{H}}$ given in Table 1 ; see section 3 ), positrons diffuse over distances $\sim(80-1000)$ pc. Thus chaotic motions appear to "confine" low-energy positrons in the hot medium rather efficiently compared to collisions and anisotropic MHD turbulence.

Large-scale MHD compressible fluctuations also provide stochastic acceleration. The rate of re-acceleration is given by $\Gamma_{\text {acc }} \sim v_{\text {rms }} / 9 L$ in the limit $v_{\text {rms }} L>v \lambda_{\mathrm{mfp}}$, where $v$ is the positron velocity and $\lambda_{\mathrm{mfp}}$ its mean free-path controlled by pitch-angle scattering (Bykov 2008, private communication). Re-acceleration is efficient only if the re-acceleration rate is large compared to the Coulomb energy loss rate (see Eq. (23)), i.e., if $v_{\text {rms }}>\left(80 \mathrm{~km} \mathrm{~s}^{-1}\right)(L / 10 \mathrm{pc}) n_{\mathrm{e}, \mathrm{cm}^{-3}} \beta^{-1}$. Re-acceleration can contribute to delaying the annihilation of positrons.

At the scales explored by the gyromotion of positrons produced by radioactive decay or by cosmic-ray interactions, one should also account for transport processes similar to those invoked for low-energy particles in the solar wind. Ragot (1999), for instance, considered the role of non-resonant fast magnetosonic waves in the dissipation regime of turbulence, i.e., above the steepening observed at a fraction of the thermal proton gyrofrequency (see Alexandrova et al. 2008, and references therein). This non-resonant transport process can produce efficient angular scattering through pitch angles $\alpha \rightarrow \pi / 2$ and thus govern the particle mean free-path at energies $\sim 1 \mathrm{MeV}$. We believe that this process adapted to the stellospheres of massive stars (typically a few parsecs in size) should be relevant for the problem of positron transport.

A full investigation of the local transport of positrons within the stellospheres of massive stars and of their spatial transport and re-acceleration in chaotic compressible turbulence is beyond the scope of this paper and deserves separate future studies.

\subsection{Galactic winds: large-scale advection}

Galactic winds can produce a systematic shift of the fluid at velocity $V_{\mathrm{w}}$ toward high Galactic latitudes. Positrons coupled to the fluid will be transported over their lifetimes $\tau_{\text {life }}$ over typical distances $\sim V_{\mathrm{w}} \tau_{\text {life }}$ (for a constant wind velocity). As mentioned above, large-scale winds can be driven by the collective action of massive stars (stellar winds and supernova explosions) in active regions of the Galaxy or by the streaming of cosmic rays into the Galactic halo.

Several observations support the idea of starburst episodes in the history of our Galaxy (some of their properties were reviewed by Veilleux et al. 2005). There are further pieces of evidence indicating an active nuclear wind in our Galaxy. For instance, powerful mass ejections from the Galactic center were observed on scales from a few arcminutes to tens of degrees at several wavelengths (Morris \& Serabyn 1996; YusefZadeh et al. 2000). Some evidence for a large-scale bipolar wind from the Galactic center was found in infrared dust emission (Bland-Hawthorn \& Cohen 2003). The authors attributed their $8.3 \mu \mathrm{m}$ observations to dust entrained in a Galactic wind that was powered by a central starburst several million years ago. Wind velocities in the Galactic center are difficult to estimate. Bland-Hawthorn \& Cohen (2003) considered vertical velocities $\sim 150 \mathrm{~km} \mathrm{~s}^{-1}$ at Galactic heights of a few $100 \mathrm{pc}$. A recent HST and FUSE data analysis (Keeney et al. 2006) uncovered four high-velocity absorption components with typical velocities $\sim 250 \mathrm{~km} \mathrm{~s}^{-1}$ at a distance of a few kpc from the Galactic plane. One of these components was blueshifted and interpreted as being part of a Galactic fountain returning matter toward the Galactic center. Alternatively, these observations might correspond to wind velocities at high altitudes ( $\gtrsim 1 \mathrm{kpc})$ that would be driven by the combined effect of the flux of cosmic rays escaping into the halo and the pressure of the thermal plasma (Everett et al. 2008).

The wind closer to the Galactic disk or the Galactic center is certainly slower, and it probably speeds up with height. Totani (2006), instead of a starburst episode, favored a wind with velocity $\sim 100 \mathrm{~km} \mathrm{~s}^{-1}$ produced by flaring activity from Sgr A*. Assuming convective transport (no other type of transport was considered in his analysis) throughout the lifetimes of positrons makes it possible to fill the extended $511 \mathrm{keV}$ bulge.

Another constraint on wind velocities, probably more relevant to positrons produced in the Galactic disk, comes from the presence of cosmic-ray radioactive elements and the analysis of secondary-to-primary ratios. Incorporated into a transport model, these observables are best fitted with a wind of constant velocity $\lesssim 15 \mathrm{~km} \mathrm{~s}^{-1}$ (Strong et al. 2007; Maurin et al. 2002). Under the assumption of a constant wind velocity of $15 \mathrm{~km} \mathrm{~s}^{-1}$ and for typical positron lifetimes $\sim\left(10^{5} \mathrm{yr}\right) / n_{\mathrm{H}, \mathrm{cm}^{-3}}$ (see Sect. 3), the distances covered by convection only are rather modest $\left(\sim(1.5 \mathrm{pc}) / n_{\mathrm{H}, \mathrm{cm}^{-3}}\right)$. We do not expect this effect to be dominant in the transport of positrons from the Galactic disk. 


\section{Conclusion}

The purpose of this work was to carefully examine the propagation of positrons with kinetic energies $\lesssim 10 \mathrm{MeV}$ in the different phases of the ISM. We identified and analyzed three main transport mechanisms: scattering off MHD waves, ballistic motion along magnetic field lines and advection with large-scale fluid motion.

In Sect. 2, we presented an investigation of the scattering off of positrons by MHD waves. Two necessary conditions are required for this process to work: (1) MHD waves and positron motions must satisfy a synchrotron resonance condition and (2) the corresponding MHD waves must exist. Since MHD waves exist only at frequencies lower than the local proton cyclotron frequency, the resonance condition and, therefore, the scattering off MHD waves are restricted to positrons with kinetic energies higher than a threshold which depends on the physical parameters of the local ISM (see Table 1). We showed that positrons in the neutral phases (i.e., WNM, CM and MM) do not scatter off MHD waves, because the latter are damped by collisional effects, causing MHD cascades to be truncated at scales several orders of magnitude above the Larmor radii of $\mathrm{MeV}$ positrons. In the ionized phases, Landau damping cuts off the Alfvén cascade at the dissipation scale. In principle, positrons could be in resonance with this part of the MHD cascade. However, the anisotropy of magnetic perturbations in the ISM leaves only a small fraction of the turbulent energy injected at large scales, thereby casting some doubt on this conclusion.

We also briefly discussed the possible action of resonant Alfvén waves generated by the streaming of cosmic rays in the WIM. It appears that positrons can resonate with these waves over a rather restricted momentum range. In the case of selfgenerated streaming modes, the issue is uncertain: while the waves are probably rapidly damped in a neutral medium, the interaction of positrons with the waves injected in an ionized medium may well help to confine them near their sources over longer timescales.

We also addressed the effect of compressional magnetosonic waves produced either in the large-scale cascade or injected locally, as in the solar wind. These waves can indeed produce some particle transport and re-acceleration, especially in the HIM. The importance of re-acceleration requires a kinetic treatement of the transport problem, i.e., solving a diffusion-convection equation.

Positrons that do not scatter off MHD waves move along magnetic field lines with helical trajectories which are perturbed by collisions with the neutral and/or charged particles of the ISM. A detailed analysis of this propagation mode, also called "collisional regime", is presented in Sect. 3. We found that the pitch angle of positrons is only slightly perturbed by collisions during their propagation at high energy. The spatial distribution of positrons at the end of their lifetimes was calculated using a Monte-Carlo method, assuming that positrons are isotropically emitted at a point source in the different ISM phases, with various characteristics of the turbulent magnetic field. The spatial distributions of high-energy positrons are nearly uniform along the mean magnetic field, but with an extent $\sim 1.7$ times smaller than twice the maximum distance traveled by positrons. Turbulence induces a scattering of positrons perpendicular to the mean magnetic field, which cause an extent of the transverse distributions that is generally negligible compared to the extent of the field-aligned distributions, except for positrons at low energy ( $E \lesssim 10 \mathrm{keV}$ ) or in high-density media (e.g., in the MM). The distances traveled by thermal positrons before they annihilate are negligible compared to the distances traveled at high energy, except in the HIM. There, their lifetimes are sufficiently long to allow them to diffuse along field lines over distances larger than the typical size of the HIM.

In this collisional regime, positrons can travel over long distances (e.g., $\$ 30 \mathrm{kpc} / n_{\mathrm{H}, \mathrm{cm}^{-3}}$ for $E_{0}=1 \mathrm{MeV}$ ). Consequently, they go through several phases of the ISM before annihilating. In this case, since positrons probably propagate along field lines, the spatial distribution of their annihilation sites should depend on the spatial distribution of the Galactic magnetic field.

Finally, in Sect. 4, we reviewed the process of transport by large-scale fluid motion. There, positrons are coupled to magnetic field lines, which are frozen to the plasma. We distinguished between advection by star-driven motions and by Galactic winds. In the first case, positrons experience the turbulent motions of the hot plasma in the winds of massive stars and in supernova remnants. We estimated a diffusion coefficient $\sim 3 \times 10^{25-27} \mathrm{~cm}^{2} \mathrm{~s}^{-1}$, depending on the turbulent velocity and the scale of turbulent eddies. In the second case, positrons are transported towards high Galactic latitudes by the Galactic wind, over distances $\sim V_{w} \tau_{\text {life }}$. The Galactic wind velocity ranges from $\lesssim 15 \mathrm{~km} \mathrm{~s}^{-1}$ in the Galactic disk to $\sim 200 \mathrm{~km} \mathrm{~s}^{-1}$ a few kpc above the Galactic plane.

If the anisotropy of the magnetic field does not affect the scattering off MHD waves in the WIM and HIM, the distances traveled by positrons scattered off MHD waves have to be compared with those resulting from collisions with interstellar matter; the propagation regime leading to the shorter distances is the dominant one. Transport by winds will further increase the extent of the spatial distributions of positrons. Comparing the distances traveled by positrons, we find that scattering off MHD waves is more efficient to confine Galactic positrons. For instance, assuming quasi-linear diffusion, we estimate that $90 \%$ of the $1 \mathrm{MeV}$ positrons emitted in the WIM by a point source would fill a sphere with radius $\sim 20-77$ pc before being excluded from the resonance condition at an energy $\sim 2.5-9.5 \mathrm{keV}$ (see Table 1), depending on the true density and the maximum turbulent scale of the WIM. Low-energy positrons would then propagate along field lines in the collisional regime before annihilating. Ultimately, the spatial distribution of these positrons would have an extent ranging from $\sim 40$ to $160 \mathrm{pc}$, i.e., lower than or similar to the typical size of the WIM. This implies that all or a large fraction of these positrons would annihilate in the WIM, where they are produced. The result is quite different in the case of anisotropic turbulence. If we neglect the effect of scattering off MHD waves in the WIM, then the spatial distribution of positrons would extend between $\sim 33 \mathrm{kpc}$ and $100 \mathrm{kpc}$ (see Fig. 8), which implies that $1 \mathrm{MeV}$ positrons systematically escape from the WIM.

The effect of the confinement of positrons close to their sources has to be taken into account in future investigations of their origin. The media in which positrons are likely to be produced are generally turbulent (supernova envelopes, supernova remnants, jets, winds). This effect would not only delay the time when positrons escape in the ISM, but would also lower their "initial" kinetic energy when they are released in the ISM. Therefore, such positrons would not propagate too far from their birth sites and the spatial distribution of sources would be closely related to the spatial distribution of the annihilation emission. Such a scenario is in favor of the hypothesis that LMXBs are a dominant source of positrons in the Galactic disk. Indeed, since these sources are expected to release low-energy positrons through winds and/or jets (Guessoum et al. 2006), they may explain that the longitudinal asymmetry of the annihilation emission observed in the Galactic disk fits in with the asymmetry 
observed in the distribution of LMXBs emitting at high energy (Weidenspointner et al. 2008). This requires that positrons propagate to distances shorter than a few kpc from their sources in the Galactic disk, in order to preserve the asymmetry, i.e., that their initial kinetic energies are lower than a few hundred $\mathrm{keV}$ if they propagate in the warm media in the collisional regime.

To conclude, we showed that the propagation of positrons in our Galaxy depends on their initial kinetic energy and on the physical conditions of the ISM in which they propagate. Therefore, in order to solve the mystery of the origin of Galactic positrons, a careful study and modelling of their sources (spatial distribution in our Galaxy, initial kinetic energy,...) and their propagation (ambient medium, mean magnetic field, turbulence,...) have to be undertaken. Here, we recall that the physical parameters of the different ISM phases used in this study are characteristic of the Galactic disk. The physical parameters are different in the Galactic bulge and particularly close to Sgr A*. A detailed calculation for each kind of source will be presented in a future paper. The results of such calculations will be compared to the spatial and spectral distributions of the annihilation emission measured by SPI.

Acknowledgements. The authors would like to thank M. Lee for his helpful comments, and A. Bykov for many enlighting discussions. The authors also thank the anonymous referee for helpful corrections and comments. This work was partially supported by the the ISSI (International Space Science Institute at Bern) programme for International Team work (ID \#110 on "Positron annihilation in the Galaxy").

\section{Appendix A: MHD wave cascade damping}

\section{A.1. Collisional damping}

Alfvén waves

\section{a. Ionized phases}

With the parameter values listed in Table 1, the proton collisional mean free-path (Eq. (16)) is $\lambda_{\mathrm{p}} \simeq(0.8-1.6) \times 10^{18} \mathrm{~cm}$ in the hot phase and $\lambda_{\mathrm{p}} \simeq(1.6-5.8) \times 10^{12} \mathrm{~cm}$ in the warm ionized phase. Clearly, $L_{\text {inj }} \gg \lambda_{\mathrm{p}} \gg k_{\| \max }^{-1}$ in both media.

In the collisional range $L_{\mathrm{inj}} \geq k^{-1}>\lambda_{\mathrm{p}}$, the main damping mechanism of Alfvén waves is viscous damping (e.g., Lazar et al. 2003), which proceeds at a rate

$\Gamma_{\text {visc }}=\frac{1}{2} \frac{\eta_{1}}{\rho_{\mathrm{i}}} k^{2}\left(\sin ^{2} \Theta+4 \cos ^{2} \Theta\right)$,

where

$\eta_{1}=0.3 \frac{n_{\mathrm{i}} k_{\mathrm{B}} T_{\mathrm{i}}}{\Omega_{\mathrm{ci}}^{2} \tau_{\mathrm{i}}}+0.51 \frac{n_{\mathrm{e}} k_{\mathrm{B}} T_{\mathrm{e}}}{\Omega_{\mathrm{ce}}^{2} \tau_{\mathrm{e}}}$

is the (ion + electron) viscosity coefficient (Braginskii 1965; note that we divided his damping rate, which applies to the wave energy, by a factor of 2 so as to obtain the amplitude damping rate). When $T_{\mathrm{i}} \simeq T_{\mathrm{e}}$, as will be assumed here, the electron contribution to the viscosity coefficient is negligible. It then follows from Eqs. (14) and (A.1) that

$\Gamma_{\text {visc }} \tau_{\mathrm{A}} \leq 0.2 \frac{1}{\left(\Omega_{\mathrm{ci}} \tau_{\mathrm{i}}\right)^{2}} \frac{v_{\mathrm{i}}}{V_{\mathrm{A}}}\left(\lambda_{\mathrm{p}} k\right) \frac{1}{\cos \Theta}$.

In a fully ionized pure-hydrogen gas, the ion rms velocity, $v_{\mathrm{i}}=\sqrt{3 k_{\mathrm{B}} T_{\mathrm{i}} / m_{\mathrm{i}}}$, is approximately equal to the adiabatic sound speed, $C_{\mathrm{s}}=\sqrt{\gamma P / \rho}$. The latter is everywhere smaller than or on the order of the Alfvén speed, $V_{\mathrm{A}}$ - more specifically, $C_{\mathrm{s}}<V_{\mathrm{A}}$ everywhere, except in the hot low- $B$ phase, where $C_{\mathrm{s}} \simeq(2.7-3.8) V_{\mathrm{A}}$. Since, in addition, $\Omega_{\mathrm{ci}} \tau_{\mathrm{i}} \gg 1$, one has $\Gamma_{\text {visc }} \tau_{\mathrm{A}} \lll 1$ throughout the collisional range (except in the limit $\cos \Theta \rightarrow 0$, which is of little interest here), so that the Alfvén wave cascade proceeds virtually undamped down to $\lambda_{\mathrm{p}}$.

b. Atomic phases

In the weakly ionized (warm and cold) atomic phases, Alfvén waves are damped by ion-neutral collisions (Kulsrud $\&$ Pearce 1969). Near the injection scale, Alfvén waves have $\omega=V_{\mathrm{A} \text {,tot }} k_{\|} \ll v_{\mathrm{in}}, v_{\mathrm{ni}}$, as can be verified with the help of the relations $v_{\mathrm{in}} \simeq\left(1.6 \times 10^{-9} \mathrm{~cm}^{3} \mathrm{~s}^{-1}\right) n_{\mathrm{n}}$ and $v_{\mathrm{ni}} \simeq\left(1.6 \times 10^{-9} \mathrm{~cm}^{3} \mathrm{~s}^{-1}\right) n_{\mathrm{i}}($ see section 2.1$)$, together with $V_{\mathrm{A}, \text { tot }}=B / \sqrt{4 \pi m_{\mathrm{p}} n_{\mathrm{H}}}, k_{\|} \lesssim 1 / L_{\mathrm{inj}}$, and thus $V_{\mathrm{A}, \text { tot }} k_{\|} \lesssim$ $\left(7.1 \times 10^{-16} \mathrm{~s}^{-1}\right)\left(B_{\mu \mathrm{G}} / \sqrt{n_{\mathrm{H}, \mathrm{cm}^{-3}}}\right)$. Again, the relevant parameter values can be found in Table 1 . In this low-frequency regime, the damping rate due to ion-neutral collisions is given by

$\Gamma_{\text {in }}=\frac{V_{\mathrm{A}, \text { tot }}^{2} k_{\|}^{2}}{2 v_{\mathrm{ni}}}$

(Ferriere et al. 1988).

Introducing Eqs. (A.4) and (14) with $V_{\mathrm{A}}=V_{\mathrm{A} \text {,tot }}$ into Eq. (17) then yields for the cutoff parallel wavenumber

$k_{\| \mathrm{cut}}=\frac{2 v_{\mathrm{ni}}}{V_{\mathrm{A}, \mathrm{tot}}}=\left(1.5 \times 10^{-14} \mathrm{~cm}^{-1}\right) \frac{n_{\mathrm{i}, \mathrm{cm}^{-3}} \sqrt{n_{\mathrm{H}, \mathrm{cm}^{-3}}}}{B_{\mu \mathrm{G}}}$.

Clearly, this expression is such that $V_{\mathrm{A} \text {,tot }} k_{\|} \sim v_{\mathrm{ni}} \ll v_{\mathrm{in}}$, which is not quite inside, but not too far from the validity limit of Eq. (A.4).

A comparison with the right-hand side of Eq. (12) immediately shows that $k_{\| \text {cut }} \ll k_{\| \max }$, which means that the Alfvén wave cascade is cut off by ion-neutral collisions way before reaching the maximum parallel wavenumber. Moreover, since positrons can interact resonantly with Alfvén waves only over a restricted energy range just above $E_{\mathrm{k}, \min }$, corresponding to a restricted wavenumber range just below $k_{\| \max }$ (see Sect. 2.1), we may conclude that the Alfvén wave cascade will produce no waves capable of resonantly interacting with positrons in the (warm and cold) atomic phases of the ISM.

c. Molecular medium

In molecular clouds, Alfvén waves are again damped by ion-neutral collisions. Including the effect of collisions of gas particles on grains, Elmegreen \& Fiebig (1993) accurately calculated the minimum scale of Alfvén waves in a molecular cloud of radius $R$. They found that the maximum value of the product $R k$ is on the order of a few for a typical cloud density $\sim 10^{4} \mathrm{~cm}^{-3}$. Since $R$ typically ranges between 0.01 and $10 \mathrm{pc}$, this leads to a cutoff wavenumber $k_{\text {cut }} \sim\left(10^{-19}-10^{-16}\right) \mathrm{cm}^{-1}$. Here, too, the smallest scale of the Alfvén wave cascade is considerably larger than the scales at which resonant interactions with positrons occur.

\section{Fast magnetosonic waves}

a. Ionized phases

Like Alfvén waves, magnetosonic waves with $L_{\mathrm{inj}} \geq k^{-1}>$ $\lambda_{\mathrm{p}}$ are primarily damped by viscous friction ${ }^{4}$. However, because of their compressible nature, they decay away much

${ }^{4}$ We checked that Joule damping leads to a cutoff wavenumber several orders of magnitude greater than $\lambda_{\mathrm{p}}^{-1}$. It only dominates viscous damping at propagation angles $\Theta \rightarrow 0$. 
faster than Alfvén waves - by a factor $\sim\left(\Omega_{\mathrm{ci}} \tau_{\mathrm{i}}\right)^{2}$. The correct expression of their viscous damping rate reads

$\Gamma_{\text {visc }}=\frac{1}{6} \frac{\eta_{0}}{\rho_{\mathrm{i}}} k^{2} \sin ^{2} \Theta+\left(\Gamma_{\mathrm{visc}}\right)_{\mathrm{A}}$,

where

$\eta_{0}=0.96 n_{\mathrm{i}} k_{\mathrm{B}} T_{\mathrm{i}} \tau_{\mathrm{i}}+0.73 n_{\mathrm{e}} k_{\mathrm{B}} T_{\mathrm{e}} \tau_{\mathrm{e}}$

is the (ion + electron) viscosity coefficient and $\left(\Gamma_{\text {visc }}\right)_{\mathrm{A}}$ denotes the viscous damping rate of Alfvén waves (given by Eq. (A.1)) (Braginskii 1965). The latter is negligible, except in the limit $\sin \Theta \rightarrow 0$.

With both $\left(\Gamma_{\text {visc }}\right)_{\mathrm{A}}$ and the electron contribution to $\eta_{0}$ neglected, Eqs. (15) and (A.6) combine to give

$\Gamma_{\text {visc }} \tau_{\mathrm{F}}=0.0533 \frac{v_{\mathrm{i}}}{V_{\mathrm{ms}}} \lambda_{\mathrm{p}} L_{\text {inj }}^{1 / 2} k^{3 / 2} \sin ^{2} \Theta$.

As mentioned below Eq. (A.3), $v_{\mathrm{i}} \simeq C_{\mathrm{s}}$, so that $v_{\mathrm{i}} / V_{\mathrm{ms}}<1$. It then follows that, at the injection scale $\left(k=L_{\text {inj }}^{-1}\right)$, $\Gamma_{\text {visc }} \tau_{\mathrm{F}}<1$.

At the transition between the collisional and collisionless ranges $\left(k=\lambda_{\mathrm{p}}^{-1}\right)$,

$\left[\Gamma_{\text {visc }} \tau_{\mathrm{F}}\right]_{\lambda_{\mathrm{p}}^{-1}}=0.0533 \frac{v_{\mathrm{i}}}{V_{\mathrm{ms}}}\left(\frac{L_{\mathrm{inj}}}{\lambda_{\mathrm{p}}}\right)^{1 / 2} \sin ^{2} \Theta$,

which can be either smaller or larger than unity, depending on the considered ISM phase and on the propagation angle, $\Theta$.

In the hot phase, $\left[\Gamma_{\mathrm{visc}} \tau_{\mathrm{F}}\right]_{\lambda_{\mathrm{p}}^{-1}}<1$ at all propagation angles. This means that the entire fast magnetosonic cascade manages to reach the collisionless range beyond $\lambda_{\mathrm{p}}^{-1}$, with only little or moderate damping.

In contrast, in the warm ionized phase, $\left[\Gamma_{\text {visc }} \tau_{\mathrm{F}}\right]_{\lambda_{\mathrm{p}}^{-1}}<1$ only for $\Theta<\Theta_{c}$, with $\Theta_{c} \simeq 2.60^{\circ}-4.15^{\circ}$. If all the waves preserve their propagation angles across the cascade, then waves with $\Theta<\Theta_{\text {c }}$ reach the collisionless range, whereas those with $\Theta>\Theta_{\mathrm{c}}$ are viscous-damped before reaching $\lambda_{\mathrm{p}}^{-1}$, and their cutoff wavenumber, given by Eq. (18), is a decreasing function of $\Theta$ :

$k_{\text {cut }}=7.06\left(\frac{V_{\mathrm{ms}}}{v_{\mathrm{i}}}\right)^{2 / 3} \lambda_{\mathrm{p}}^{-2 / 3} L_{\mathrm{inj}}^{-1 / 3}(\sin \Theta)^{-4 / 3}$,

or, numerically, $k_{\text {cut }} \simeq\left((0.5-1.0) \times 10^{-14} \mathrm{~cm}^{-1}\right)(\sin \Theta)^{-4 / 3}$. However, the actual situation may not be as clear-cut, as in reality, the energy transfer down the cascade is accompanied by a randomization of $\Theta$, due to both nonlinear interactions between modes with non-parallel wave vectors and wandering of magnetic field lines. Yan \& Lazarian (2004) estimated that the variation in $\Theta$ is $\delta \Theta \sim\left(k L_{\text {inj }}\right)^{-1 / 4}$. At $k \sim 10^{-14} \mathrm{~cm}^{-1}$, this gives $\delta \Theta \sim 0.02$, which is small, but not negligible compared to the small values of $\Theta_{c}$. Ultimately, we cannot rule out the possibility that even waves with $\Theta<\Theta_{c}$ are cut off by viscous damping before reaching $\lambda_{\mathrm{p}}^{-1}$.

b. Atomic phases

Here, fast magnetosonic waves are damped by ion-neutral collisions, at a rate

$\Gamma_{\mathrm{in}}=\frac{V_{\mathrm{A}, \mathrm{tot}}^{2} k^{2}}{2 v_{\mathrm{ni}}} f(\Theta)$, with

$$
f(\Theta)=\frac{1}{2}\left(1+\frac{\left|V_{\mathrm{A}, \text { tot }}^{2}-C_{\mathrm{s}}^{2}\right|}{\sqrt{V_{\mathrm{A}, \text { tot }}^{4}+C_{\mathrm{s}}^{4}-2 V_{\mathrm{A}, \text { tot }}^{2} C_{\mathrm{s}}^{2} \cos 2 \Theta}}\right)
$$

(Ferriere et al. 1988).

Substitution of Eqs. (A.10) and (15) into Eq. (18) directly gives for the cutoff wavenumber

$k_{\mathrm{cut}}=\left(\frac{2 v_{\mathrm{ni}}}{V_{\mathrm{A}, \mathrm{tot}}^{2}} V_{\mathrm{ms}} L_{\mathrm{inj}}^{-1 / 2} \frac{1}{f(\Theta)}\right)^{2 / 3}$.

Since $V_{\mathrm{A}, \text { tot }}>C_{\mathrm{s}}$ in both atomic phases, we may, to the order of the present approximation, let $V_{\mathrm{ms}} \simeq V_{\mathrm{A} \text {,tot }}$ and $f(\Theta) \simeq 1$ in the above equation, whereupon we obtain

$$
\begin{aligned}
k_{\mathrm{cut}} & \simeq\left(\frac{2 v_{\mathrm{ni}}}{V_{\mathrm{A}, \mathrm{tot}}}\right)^{2 / 3} L_{\mathrm{inj}}^{-1 / 3} \\
& \simeq\left(0.9 \times 10^{-16} \mathrm{~cm}^{-1}\right)\left(\frac{n_{\mathrm{i}, \mathrm{cm}}{ }^{-3} \sqrt{n_{\mathrm{H}, \mathrm{cm}^{-3}}}}{B_{\mu \mathrm{G}}}\right)^{2 / 3} .
\end{aligned}
$$

The fast magnetosonic wave cascade is cut off by ion-neutral collisions way before reaching the small scales at which positrons can be in resonant interaction.

c. Molecular medium

Our conclusion is similar to that reached for Alfvén waves.

\section{A.2. Collisionless damping}

In Sect. A.1, we saw that, in the ionized phases of the ISM, the Alfvén wave cascade experiences negligible collisional damping, which enables it to make it all the way down to the collisionless range $k^{-1}<\lambda_{\mathrm{p}}$. The fast magnetosonic wave cascade, in contrast, experiences significant collisional (viscous) damping. In the hot ionized phase, the collisional range $L_{\text {inj }} \geq k^{-1}>\lambda_{\mathrm{p}}$ is sufficiently narrow that the fast magnetosonic cascade nevertheless reaches the collisionless range only partially attenuated. But in the warm ionized phase, where the collisional range spans roughly eight decades, the fast magnetosonic cascade completely (or almost completely) decays away before reaching the collisionless range.

We now examine collisionless damping in the cases of interest, namely, for the Alfvén cascade in the hot and warm ionized phases and for the fast magnetosonic cascade in the hot ionized phase. ${ }^{5}$ In all cases, the dominant collisionless damping mechanism is linear Landau damping.

\section{Alfvén waves}

For Alfvén waves, an approximate expression of the linear Landau damping rate is (Ginzburg 1961):

$$
\begin{aligned}
\Gamma_{\mathrm{LD}}= & \sqrt{\frac{\pi}{8}} v_{\mathrm{e}}^{\prime} \frac{k^{3}}{k_{\mathrm{A}}^{2}} \frac{\cos \Theta \sin ^{2} \Theta}{\sin ^{2} \Theta+3 \frac{k^{2}}{k_{\mathrm{A}}^{2}} \cos ^{4} \Theta} \\
& \times\left[\frac{v_{\mathrm{i}}^{\prime 2}}{v_{\mathrm{e}}^{\prime 2}}+\left(\sin ^{2} \Theta+4 \cos ^{2} \Theta\right) \exp \left(-\frac{V_{\mathrm{A}}^{2}}{2 v_{\mathrm{i}}^{\prime 2}}\right)\right],
\end{aligned}
$$

\footnotetext{
5 We will, however, keep in mind the possibility that, in the warm ionized phase, waves with small propagation angles might enter the collisionless regime.
} 
where $v_{\mathrm{i}}^{\prime}=\sqrt{k_{\mathrm{B}} T_{\mathrm{i}} / m_{\mathrm{i}}}$ and $v_{\mathrm{e}}^{\prime}=\sqrt{k_{\mathrm{B}} T_{\mathrm{e}} / m_{\mathrm{e}}}$ are the ion and electron thermal speeds (which differ from the ion and electron rms velocities, $v_{\mathrm{i}}$ and $v_{\mathrm{e}}$, by a factor $\sqrt{3}$ ) and $k_{\mathrm{A}}=\Omega_{\mathrm{ci}} / V_{\mathrm{A}}$ is the inverse ion inertial length. For a pure-hydrogen plasma, $k_{\mathrm{A}}$ is the maximum parallel wavenumber, $k_{\| \max }$ (see Eq. (6)).

Multiplying Eq. (A.13) by Eq. (14) leads to

$$
\begin{aligned}
\Gamma_{\mathrm{LD}} \tau_{\mathrm{A}}= & \sqrt{\frac{\pi}{8}} \frac{v_{\mathrm{e}}^{\prime}}{V_{\mathrm{A}}} \frac{k^{2}}{k_{\mathrm{A}}^{2}} \frac{\sin ^{2} \Theta}{\sin ^{2} \Theta+3 \frac{k^{2}}{k_{\mathrm{A}}^{2}} \cos ^{4} \Theta} \\
& \times\left[\frac{v_{\mathrm{i}}^{\prime 2}}{v_{\mathrm{e}}^{\prime 2}}+\left(\sin ^{2} \Theta+4 \cos ^{2} \Theta\right) \exp \left(-\frac{V_{\mathrm{A}}^{2}}{2 v_{\mathrm{i}}^{\prime 2}}\right)\right] .
\end{aligned}
$$

The cutoff of the Alfvén cascade occurs when $\Gamma_{\mathrm{LD}} \tau_{\mathrm{A}}=1$ (see Eq. (17)), provided that this relation admits a real solution for $k$.

In the hot low- $B$ and warm ionized phases, where $V_{\mathrm{A}} \lesssim v_{\mathrm{i}}^{\prime}$, the first term inside the square brackets on the right-hand side of Eq. (A.14) is negligible, and the relation $\Gamma_{\mathrm{LD}} \tau_{\mathrm{A}}=1$ admits one solution, given by

$$
\begin{aligned}
k_{\text {cut }} \simeq & \left(\frac{8}{\pi}\right)^{1 / 4}\left(\frac{V_{\mathrm{A}}}{v_{\mathrm{e}}^{\prime}}\right)^{1 / 2} \\
& \times \exp \left(\frac{V_{\mathrm{A}}^{2}}{4 v_{\mathrm{i}}^{\prime 2}}\right)\left(\sin ^{2} \Theta+4 \cos ^{2} \Theta\right)^{-1 / 2} k_{\mathrm{A}}
\end{aligned}
$$

(except in the limit $\sin \Theta \rightarrow 0$ ). Ignoring the weak $\Theta$-dependence of $k_{\text {cut }}$ (which entails only a variation by a factor of 2 ), we find $k_{\text {cut }} \simeq(0.14-0.18) k_{\mathrm{A}}$ in the hot low- $B$ phase and $k_{\text {cut }} \simeq$ $(0.65-3.20) k_{\mathrm{A}}$ in the warm ionized phase. Hence, in these two media, the Alfvén cascade is cut off by Landau damping at a scale close to the proton inertial length.

In the hot high- $B$ phase, where $V_{\mathrm{A}}$ exceeds $v_{\mathrm{i}}^{\prime}$ by a factor $\simeq 4.8-6.8$, the exponential factor on the right-hand side of Eq. (A.14) becomes negligibly small, with the result that $\Gamma_{\mathrm{LD}} \tau_{\mathrm{A}}<1$ at all $k$ (except in the limit $\cos \Theta \rightarrow 0$, but in this limit Alfvén waves are viscous-damped before reaching the collisionless range; see Eq. (A.3)). If taken at face value, this result would lead to the erroneous conclusion that the Alfvén cascade is not cut off by Landau damping.

The truth is that our expressions for the frequency, transfer time and damping rate of Alfvén waves are all strictly valid only in the MHD regime, so that Eq. (A.14) actually breaks down at wavenumbers approaching the inverse proton inertial length, $k_{\mathrm{A}}$. The only conclusion that can be drawn from Eq. (A.14) is that the cutoff of the Alfvén cascade by Landau damping occurs roughly at the inverse proton inertial length, $k_{\mathrm{A}}$.

\section{Fast magnetosonic waves}

For fast magnetosonic waves, linear Landau damping proceeds at a rate (Ginzburg 1961)

$\Gamma_{\mathrm{LD}}=\sqrt{\frac{\pi}{8}} v_{\mathrm{i}}^{\prime} k \frac{\sin ^{2} \Theta}{\cos \Theta}\left[\frac{v_{\mathrm{i}}^{\prime}}{v_{\mathrm{e}}^{\prime}}+5 \exp \left(-\frac{V_{\mathrm{F}}^{2}}{2 v_{\mathrm{i}}^{\prime 2} \cos ^{2} \Theta}\right)\right]$,

which, combined with Eq. (15), yields

$$
\begin{aligned}
\Gamma_{\mathrm{LD}} \tau_{\mathrm{F}}= & \sqrt{\frac{\pi}{8}} \frac{v_{\mathrm{i}}^{\prime}}{V_{\mathrm{ms}}}\left(k L_{\mathrm{inj}}\right)^{1 / 2} \frac{\sin ^{2} \Theta}{\cos \Theta} \\
& \times\left[\frac{v_{\mathrm{i}}^{\prime}}{v_{\mathrm{e}}^{\prime}}+5 \exp \left(-\frac{V_{\mathrm{F}}^{2}}{2 v_{\mathrm{i}}^{\prime 2} \cos ^{2} \Theta}\right)\right] .
\end{aligned}
$$

The cutoff wavenumber, at which $\Gamma_{\mathrm{LD}} \tau_{\mathrm{F}}=1$ (see Eq. (18)), is given by

$$
\begin{aligned}
k_{\text {cut }}= & \frac{8}{\pi} \frac{V_{\mathrm{ms}}^{2}}{v_{\mathrm{i}}^{\prime 2}} \frac{\cos ^{2} \Theta}{\sin ^{4} \Theta} \\
& \times\left[\frac{v_{\mathrm{i}}^{\prime}}{v_{\mathrm{e}}^{\prime}}+5 \exp \left(-\frac{V_{\mathrm{F}}^{2}}{2 v_{\mathrm{i}}^{\prime 2} \cos ^{2} \Theta}\right)\right]^{-2} L_{\text {inj }}^{-1} .
\end{aligned}
$$

Here, the cutoff depends strongly on $\Theta$, and it diverges at parallel propagation (just like the viscous cutoff in the warm ionized phase; see Eq. (A.9)). Once again, due to the possible randomization of $\Theta$ (see Sect. A.1), this mathematical divergence should not be a concern, unless the randomization process is inefficient.

The only interstellar phase that needs to be considered here is the hot ionized phase. In the low- $B$ case, $V_{\mathrm{A}}<v_{\mathrm{i}}^{\prime}$ and the expression within square brackets in Eq. (A.18) is dominated by the exponential term (except in the limit $\cos \Theta \rightarrow 0$ ). Equation (A.18) then reduces to $k_{\text {cut }} \gtrsim(0.46-0.61) L_{\text {inj }}^{-1} \cos ^{2} \Theta / \sin ^{4} \Theta$. Despite the strong $\Theta$-dependence of $k_{\text {cut }}$, we may conclude that the fast magnetosonic cascade is severely affected by Landau damping as soon as it enters the collisionless range and that it globally decays away shortly below $\lambda_{\mathrm{p}}$.

In the high- $B$ case, $V_{\mathrm{A}}>v_{\mathrm{i}}^{\prime}$, the exponential term drops out from Eq. (A.18), and the result reads $k_{\text {cut }}$ $\simeq(1.2-2.3) \times 10^{5} L_{\mathrm{inj}}^{-1} \cos ^{2} \Theta / \sin ^{4} \Theta \simeq((4.0-7.5) \times$ $\left.10^{-16} \mathrm{~cm}^{-1}\right) \cos ^{2} \Theta / \sin ^{4} \Theta$. Here, Landau damping is less severe, which allows the fast magnetosonic cascade to globally proceed over roughly three decades below $\lambda_{\mathrm{p}}$, before vanishing.

\section{Appendix B: Summary of the Monte Carlo algorithm}

This section presents a summary of the Monte-Carlo algorithm used to simulate the collisional transport of positrons in an ISM with given physical parameters. The method, the equations and the parameters are described in Sect. 3. $E_{\text {user }}$ is the initial positron kinetic energy specified by the user.

1 Load the differential and total cross sections, rate of variation of the average scattering-angle and energy-loss rates as functions of positron energy for the given ISM parameters (see Sect. 3.1.1, Eq. (26), Sect. 3.1.2 and references therein).

2 Calculate the distribution function for collisions with $\mathrm{H}$ and annihilation with $\mathrm{e}^{-}$as functions of positron energy (right hand side of Eq. (29)).

3 Initialize the kinetic parameters of the considered positron

3.1 Set $k=0, \boldsymbol{r}_{0}=(x, y, z)=(0,0,0), t_{0}=0$ and $E_{0}=E_{\text {user }}$. 3.2 Set the initial direction (isotropic):

3.2.1 $\cos \alpha_{k}$ is chosen randomly between -1 and 1 ;

3.2.2 $\phi_{k}$ is chosen randomly between 0 and $2 \pi$.

4 While the kinetic energy $E_{k}$ of the positron is larger than $100 \mathrm{eV}$ do

4.1 calculate the gyroradius and the gyrofrequency;

4.2 calculate the scattering time $\delta t_{\mathrm{s}}$ (Eq. (31));

4.3 calculate the energy $E_{\text {collision }}$ at which a collision occurs (Eq. (29));

4.4 calculate the time of flight before the collision $\delta t_{\mathrm{c}}$;

4.5 if $\delta t_{\mathrm{s}}$ is smaller than $\delta t_{\mathrm{c}}$ then $^{6}$

4.5.1 calculate the energy lost $\Delta E=\frac{\mathrm{d} E}{\mathrm{~d} t} \times \delta t_{\mathrm{s}}$;

4.5.2 calculate the average scattering angle (Eq. (25));

\footnotetext{
${ }^{6}$ The positron propagates without colliding with a neutral or annihilating with an electron.
} 
4.5.3 calculate the new pitch angle $\alpha_{k+1}$ and phase $\phi_{k+1}$ (Eqs. (27) and (28));

4.5.4 calculate the new position $\boldsymbol{r}_{k+1}$ and time $t_{k+1}=t_{k}+$ $\delta t_{\mathrm{s}}$

4.5.5 set the energy $E_{k+1}=E_{k}+\Delta E$;

4.6 Else if $\delta t_{s}$ is greater than $\delta t_{c}$ then $^{7}$

4.6.1 calculate the average scattering angle before the collision (replace $\delta t_{s}$ by $\delta t_{c}$ in Eq. (25));

4.6.2 calculate the new pitch angle $\alpha_{k+1}$ and phase $\phi_{k+1}$ before the collision (Eqs. (27) and (28));

4.6.3 calculate the new position $\boldsymbol{r}_{k+1}$ and the time $t_{k+1}=$ $t_{k}+\delta t_{\mathrm{c}}$

4.6.4 choose randomly the kind of collision (Eq. (30));

4.6.5 if the interaction is an annihilation then $E_{k+1}=0$ and go to step 5;

4.6.6 calculate the energy lost in the collision $\Delta E_{\text {collision }}$ (Sect. 3.1.2);

4.6.7 calculate the new pitch angle $\alpha_{k+1}$ and phase $\phi_{k+1}$ after the collision (Sect. 3.1.2, Eqs. (27) and (28));

4.6.8 set the energy $E_{k+1}=E_{\text {collision }}+\Delta E_{\text {collision }}$;

4.7 End of the $k$ th iteration, set $k=k+1$ and go to step 4;

5 Store the final position, time and energy and go to step 3 to process the next positron.

\section{References}

Aharonian, F., Akhperjanian, A. G., Bazer-Bachi, A. R., et al. 2006, ApJ, 636, 777

Alexandrova, O., Carbone, V., Veltri, P., \& Sorriso-Valvo, L. 2008, ApJ, 674, 1153

Armstrong, J. W., Rickett, B. J., \& Spangler, S. R. 1995, ApJ, 443, 209

Asano, K., Iwamoto, S., \& Takahara, F. 2007, ApJS, 168, 268

Beacom, J. F., \& Yüksel, H. 2006, Phys. Rev. Lett., 97, 071102

Bland-Hawthorn, J., \& Cohen, M. 2003, ApJ, 582, 246

Blumenthal, G. R. \& Gould, R. J. 1970, Rev. Mod. Phys., 42, 237

Boehm, C., Hooper, D., Silk, J., Casse, M., \& Paul, J. 2004, Phys. Rev. Lett., 92, 101301

Braginskii, S. I. 1965, Rev. Plasma Phys., 1, 205

Bussard, R. W., Ramaty, R., \& Drachman, R. J. 1979, ApJ, 228, 928

Bykov, A. M. 2001, Space Sci. Rev., 99, 317

Cardelli, J. A., Meyer, D. M., Jura, M., \& Savage, B. D. 1996, ApJ, 467, 334

Cesarsky, C. J., \& Volk, H. J. 1978, A\&A, 70, 367

Chandran, B. D. G. 2000, Phys. Rev. Lett., 85, 4656

Charlton, M., \& Humberston, J. W. 2000, Positron Physics (Cambridge, UK: Cambridge University Press)

Cheng, K. S., Chernyshov, D. O., \& Dogiel, V. A. 2006, ApJ, 645, 1138

Crutcher, R. M. 1999, ApJ, 520, 706

Dermer, C. D. 1984 , ApJ, 280, 328

Dermer, C. D. 1985, ApJ, 295, 28

Diehl, R., Prantzos, N., \& von Ballmoos, P. 2006, Nucl. Phys. A, 777, 70

Dogel, V. A. \& Sharov, G. S. 1985, Soviet Astron. Lett., 11, 346

Elmegreen, B. G., \& Fiebig, D. 1993, A\&A, 270, 397

Everett, J. E., Zweibel, E. G., Benjamin, R. A., et al. 2008, ApJ, 674, 258

Farmer, A. J., \& Goldreich, P. 2004, ApJ, 604, 671

Ferrière, K. 2001, Rev. Mod. Phys., 73, 1031

Ferriere, K. M., Zweibel, E. G., \& Shull, J. M. 1988, ApJ, 332, 984

Ferrière, K., Gillard, W., \& Jean, P. 2007, A\&A, 467, 611

Giacalone, J., \& Jokipii, J. R. 1994, ApJ, 430, L137

Ginzburg, V., L. 1961, Propagation of Electromagnetic Waves in Plasma (New York: Gordon \& Breach)

Ginzburg, V. L. 1979, Theoretical physics and astrophysics, International Series in Natural Philosophy (Oxford: Pergamon)
Goldreich, P., \& Sridhar, S. 1995, ApJ, 438, 763

Gryziński, M. 1965a, Phys. Rev., 138, 336

Gryziński, M. 1965b, Phys. Rev., 138, 305

Gryziński, M. 1965c, Phys. Rev., 138, 322

Guessoum, N., Jean, P., \& Gillard, W. 2005, A\&A, 436, 171

Guessoum, N., Jean, P., \& Prantzos, N. 2006, A\&A, 457, 753

Heiles, C., \& Troland, T. H. 2005, ApJ, 624, 773

Higdon, J. C., Lingenfelter, R. E., \& Rothschild, R. E. 2009, ApJ, 698, 350

Huba, J. D. 2006, A collection of plasma physics formulas and data - NRL plasma formulary, Tech. rep., Naval Research Laboratory

Jean, P., von Ballmoos, P., Knödlseder, J., et al. 2004, in 5th INTEGRAL

Workshop on the INTEGRAL Universe, ed. V. Schoenfelder, G. Lichti, \&

C. Winkler, ESA SP, 552, 51

Jean, P., Knödlseder, J., Gillard, W., et al. 2006, A\&A, 445, 579

Keeney, B. A., Danforth, C. W., Stocke, J. T., et al. 2006, ApJ, 646, 951

Knödlseder, J., Lonjou, V., Weidenspointner, G., et al. 2005, A\&A, 441, 513

Kulsrud, R., \& Pearce, W. P. 1969, ApJ, 156, 445

Lazar, M., Spanier, F., \& Schlickeiser, R. 2003, A\&A, 410, 415

Lazarian, A., \& Beresnyak, A. 2006, MNRAS, 373, 1195

Lerche, I. 1967, ApJ, 147, 689

Lithwick, Y., \& Goldreich, P. 2001, ApJ, 562, 279

Mac Low, M.-M. \& Klessen, R. S. 2004, Rev. Mod. Phys., 76, 125

Maurin, D., Taillet, R., \& Donato, F. 2002, A\&A, 394, 1039

McClure-Griffiths, N. M., Ford, A., Pisano, D. J., et al. 2006, ApJ, 638, 196

McCray, R., \& Kafatos, M. 1987, ApJ, 317, 190

Melrose, D. B. 1986, Instabilities in Space and Laboratory Plasmas, Instabilities in Space and Laboratory Plasmas, ed. D. B. Melrose, ISBN 0521305411 (Cambridge, UK: Cambridge University Press, August)

Morfill, G. E. 1982, MNRAS, 198, 583

Morris, M., \& Serabyn, E. 1996, ARA\&A, 34, 645

Muno, M. P., Bauer, F. E., Bandyopadhyay, R. M., \& Wang, Q. D. 2006, ApJS, 165,173

Ohno, H., \& Shibata, S. 1993, MNRAS, 262, 953

Osterbrock, D. E. 1961, ApJ, 134, 270

Parizot, E., Marcowith, A., van der Swaluw, E., Bykov, A. M., \& Tatischeff, V. 2004a, A\&A, 424, 747

Parizot, E., Marcowith, A., van der Swaluw, E., Bykov, A. M., \& Tatischeff, V. 2004b, A\&A, 424, 747

Parizot, E., Cassé, M., Lehoucq, R., \& Paul, J. 2005, A\&A, 432, 889

Prantzos, N. 2006, A\&A, 449, 869

Ptuskin, V. S., Voelk, H. J., Zirakashvili, V. N., \& Breitschwerdt, D. 1997, A\&A, 321,434

Ragot, B. R. 1999, ApJ, 518, 974

Ragot, B. R. 2006, ApJ, 642, 1163

Rand, R. J., \& Kulkarni, S. R. 1989, ApJ, 343, 760

Schlickeiser, R. \& Miller, J. A. 1998, ApJ, 492, 352

Shull, J. M., \& Draine, B. T. 1987, in Interstellar Processes, ed. D. J. Hollenbach, \& H. A. Thronson, Jr., Astrophysics and Space Science Library, 134, 283

Sizun, P., Cassé, M., \& Schanne, S. 2006, Phys. Rev. D, 74, 063514

Skilling, J. 1975, MNRAS, 173, 255

Skilling, J., \& Strong, A. W. 1976, A\&A, 53, 253

Strong, A. W., Moskalenko, I. V., \& Ptuskin, V. S. 2007, Ann. Rev. Nucl. Part. Sci., 57, 285

Toptygin, I. N. 1985, Cosmic rays in interplanetary magnetic fields, ed. I. N. Toptygin

Totani, T. 2006, PASJ, 58, 965

Veilleux, S., Cecil, G., \& Bland-Hawthorn, J. 2005, ARA\&A, 43, 769

von Ballmoos, P., Guessoum, N., Jean, P., \& Knödlseder, J. 2003, A\&A, 397, 635

Wallyn, P., Durouchoux, P., Chapuis, C., \& Leventhal, M. 1994, ApJ, 422, 610 Wang, W. 2006, Chin. J. Astron. Astrophys. Suppl., 6, 020000

Weidenspointner, G., Shrader, C. R., Knödlseder, J., et al. 2006, A\&A, 450, 1013

Weidenspointner, G., Skinner, G. K., Jean, P., et al. 2008, Nature, 451, L159

Wentzel, D. G. 1974, ARA\&A, 12, 71

Yan, H., \& Lazarian, A. 2002, Phys. Rev. Lett., 89, B1102

Yan, H., \& Lazarian, A. 2004, ApJ, 614, 757

Yusef-Zadeh, F., Melia, F., \& Wardle, M. 2000, Science, 287, 85 